\title{
Momentum maps and stochastic Clebsch action principles
}

\author{
Ana Bela Cruzeiro ${ }^{1}$, Darryl D. Holm² ${ }^{2}$ Tudor S. Ratiu ${ }^{3}$ \\ (1) GFMUL and Mathematics Department, Instituto Superior Técnico, Lisboa, Portugal \\ (2) Mathematics Department, Imperial College, London, United Kingdom \\ (3) School of Mathematics, Jiao Tong University, Shanghai, China and \\ Section de Mathématiques, Université de Genève, Switzerland \\ Version 16 October 2017 \\ Keywords: Geometric mechanics; stochastic processes; Clebsch variational principles
}

\begin{abstract}
We derive stochastic differential equations whose solutions follow the flow of a stochastic nonlinear Lie algebra operation on a configuration manifold. For this purpose, we develop a stochastic Clebsch action principle, in which the noise couples to the phase space variables through a momentum map. This special coupling simplifies the structure of the resulting stochastic Hamilton equations for the momentum map. In particular, these stochastic Hamilton equations collectivize for Hamiltonians that depend only on the momentum map variable. The Stratonovich equations are derived from the Clebsch variational principle and then converted into Itô form. In comparing the Stratonovich and Itô forms of the stochastic dynamical equations governing the components of the momentum map, we find that the Itô contraction term turns out to be a double Poisson bracket. Finally, we present the stochastic Hamiltonian formulation of the collectivized momentum map dynamics and derive the corresponding Kolmogorov forward and backward equations.
\end{abstract}

\section{Contents}

1 Background and motivation $\quad 2$

1.1 Poincaré $1901 \ldots \ldots \ldots \ldots$. . . . . . . . . . . . . . . . . . . . . . . 2

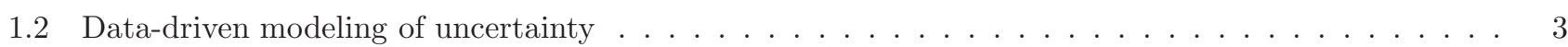

1.3 Stochastic Hamilton equations . . . . . . . . . . . . . . . . . . . . . . . 4

1.4 A distinction from other approaches . . . . . . . . . . . . . . . . . . . . . . . .

2 Variational principle for Stratonovich stochastic coadjoint motion 6

2.1 Deterministic formulation . . . . . . . . . . . . . . . . . . . . . . 6 6

2.2 Stochastic Clebsch formulation . . . . . . . . . . . . . . . . . . . . . 8

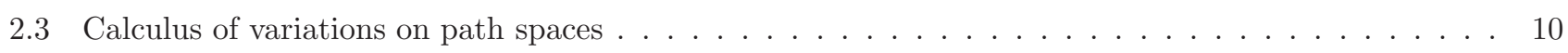

2.4 Stochastic variational principles . . . . . . . . . . . . . . . . . . . . . . 12

2.5 The stochastic equations of motion on $\mathfrak{g}^{*} \times Q \ldots \ldots \ldots$

3 Itô formulation of stochastic coadjoint motion 16 
4 Stochastic Hamiltonian formulation $\quad 20$

4.1 The deterministic Hamilton equations . . . . . . . . . . . . . . . . . . . . . . . . . . . . 20

4.2 The stochastic Hamilton equations . . . . . . . . . . . . . . . . . . . 25

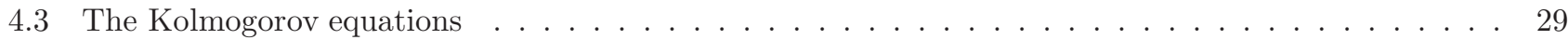

\section{Background and motivation}

\section{$1.1 \quad$ Poincaré 1901}

In 1901 Poincaré noticed that when a Lie group $G$ acts transitively on the configuration manifold $Q$ of a mechanical system, then an opportunity arises, "to cast the equations of mechanics into a new form which could be interesting to know" 27. The new form of the Euler-Lagrange equations of mechanics for a given Lagrangian $L(q, \dot{q})$ in Hamilton's principle $0=\delta \int_{a}^{b} L(q, \dot{q}) d t$ defined on the tangent bundle $T Q$ (velocity phase space) of the manifold $Q$ (state space) emerges when the motion is lifted to a set of dynamical equations for a curve $g(t) \in G$, parameterized by time, $t$, by writing the motion as $q(t)=g^{-1}(t) q_{0}$, with $g(0)=e$, the identity element of the Lie group, $G$. Let $\mathfrak{g}$ denote the Lie algebra of $G$. The Lie algebra action of the element $u:=g^{-1} \dot{g} \in \mathfrak{g}$, on the manifold $Q$ is denoted by concatenation, namely, $u q$; this is a vector field $Q \ni q \mapsto u q \in T Q$ on $Q$. Thus, if $q(t)=g^{-1}(t) q_{0}$, we have $\dot{q}(t)=-u q(t)$.

The action integral in Hamilton's principle transforms under $G$ in [27] as,

$$
\int_{a}^{b} L(q, \dot{q}) d t=: \int_{a}^{b} \tilde{L}\left(g, \dot{g} ; q_{0}\right) d t=\int_{a}^{b} \tilde{L}\left(e, g^{-1} \dot{g}, g^{-1} q_{0}\right) d t=: \int_{a}^{b} \ell\left(u, g^{-1} q_{0}\right)
$$

where $\langle\cdot, \cdot\rangle_{\mathfrak{g}}: \mathfrak{g}^{*} \times \mathfrak{g} \rightarrow \mathbb{R}$ denotes the non-degenerate pairing between the Lie algebra $\mathfrak{g}$ and its dual $\mathfrak{g}^{*}$.

Upon taking variations in Hamilton's principle, Poincaré cast the Euler-Lagrange equations for vanishing endpoint conditions into his "new form". To arrive at it, we take a deformation $g_{\varepsilon}(t)$ of the curve $g_{0}(t):=g(t)$ for $\varepsilon$ in a small interval centred at 0 , keeping the endpoints fixed, i.e., $g_{\varepsilon}(a)=g(a), g_{\varepsilon}(b)=g(b)$ for all $\varepsilon$, denote by $\delta g(t):=\left.\frac{d}{d \varepsilon}\right|_{\varepsilon=0} g_{\varepsilon}(t) \in T_{g(t)} G$, and note that $\delta g(a)=0=\delta g(b)$. Defining $v(t):=g(t)^{-1} \delta g(t) \in \mathfrak{g}$ and $\delta u(t):=\left.\frac{d}{d \varepsilon}\right|_{\varepsilon=0} g_{\varepsilon}(t)^{-1} \dot{g}_{\varepsilon}(t) \in \mathfrak{g}$, we deduce the identity $\delta u(t)=\dot{v}(t)+\operatorname{ad}_{u(t)} v(t)$, where $\operatorname{ad}_{x} y:=[x, y]$ for every $x, y \in \mathfrak{g}$ and we denote by $\operatorname{ad}_{x}^{*}: \mathfrak{g}^{*} \rightarrow \mathfrak{g}^{*}$ the dual of the linear map $\operatorname{ad}_{x}: \mathfrak{g} \rightarrow \mathfrak{g}$ for every $x \in \mathfrak{g}$. A direct computation, using (1.1), yields

$$
\int_{a}^{b}\left\langle\frac{d}{d t} \frac{\partial L}{\partial \dot{q}}-\frac{\partial L}{\partial q}, \delta q\right\rangle_{Q} d t=0=\int_{a}^{b}\left\langle\frac{d}{d t} \frac{\partial \ell}{\partial u}-\operatorname{ad}_{u}^{*} \frac{\partial \ell}{\partial u}-\mathbf{J}\left(\frac{\partial \ell}{\partial q}\right), v\right\rangle_{\mathfrak{g}} d t
$$

where $\langle\cdot, \cdot\rangle_{Q}: T^{*} Q \times T Q \rightarrow \mathbb{R}$ denotes the natural duality pairing, taken fiberwise, between the tangent bundle $T Q$ and its dual $T^{*} Q$, the phase space of the mechanical system. The map $\mathbf{J}: T^{*} Q \rightarrow \mathfrak{g}^{*}$ has the expression $\left\langle\mathbf{J}\left(p_{q}\right), w\right\rangle_{\mathfrak{g}}=\left\langle p_{q}, w q\right\rangle_{Q}$, for all $w \in \mathfrak{g}$ and is the momentum map of the cotangent lifted $G$-action on $T^{*} Q$. Thus, (1.2) yields the classical Euler-Lagrange equations, if one uses the left hand side of the identity, and it yields Poincaré's "new form" of the equations of motion, if one uses the right hand side of the identity, which is

$$
\frac{d}{d t} \frac{\partial \ell}{\partial u}=\operatorname{ad}_{u}^{*} \frac{\partial \ell}{\partial u}+\mathbf{J}\left(\frac{\partial \ell}{\partial q}\right)
$$

For more details, many applications, and reviews of the overwhelming importance of the momentum map in mechanics, see [18, 24].

In Poincaré's illustrative example, $G=S O(3)$ was the Lie group of rotations in three dimensions; the manifold $Q=\mathbb{R}^{3}$ was three dimensional Euclidean space; $\mathfrak{g}=\mathfrak{s o}(3) \simeq \mathbb{R}^{3}$ and $\mathfrak{g}^{*}=\mathfrak{s o}(3)^{*} \simeq \mathbb{R}^{3}$ were isomorphic to $\mathbb{R}^{3} ;$ the pairings $\langle\cdot, \cdot\rangle_{Q}$ and $\langle\cdot, \cdot\rangle_{\mathfrak{g}}$ were both the Euclidean scalar product; the operations ad, ad ${ }^{*}$ and the momentum map $\mathbf{J}$ were all (plus, or minus) the vector cross product in $\mathbb{R}^{3}$. Poincaré's new form of the equations of mechanics in that case reduced to Euler's equations for a heavy top. This 1901 result of Poincaré, together with Lie's discovery of the 
natural Poisson bracket on the dual of a Lie algebra, are the two key results, one in the Lagrangian, the other in the Hamiltonian formulation, of what has developed into geometric mechanics. Poincaré's paper [27] was carefully reviewed recently from a modern perspective in [23. For textbook discussions of geometric mechanics, see, e.g., [18, 24].

The aim of the present work is to continue the theme of these earlier developments by revisiting Poincaré's starting point [27] and augmenting the Lagrangian (1.1) in Hamilton's principle to introduce a Lagrange multiplier $m$ into (1.1), in preparation for introducing stochasticity later. That is, instead of (1.1), we work with the constrained action,

$$
\int_{a}^{b}\left(\ell\left(u, g^{-1} q_{0}\right)+\left\langle m, g^{-1} \dot{g}-u\right\rangle_{\mathfrak{g}}\right) d t
$$

in order to enforce the reconstruction relation $g^{-1} \dot{g}=u$ for the curve $g(t) \in G$. The reconstruction relation, in turn, generates the motion $q(t)=g^{-1}(t) q_{0}$ along a solution curve in $Q$. This constrained form of the action facilitates the introduction of stochasticity into Poincaré's original framework. That is, in following Poincaré's lead in the deterministic case, we seek to lay the framework for stochastic geometric mechanics. In deterministic geometric mechanics, as we have just seen, the time-dependent dynamics is modeled by the action of a transformation group. The geometric mechanics approach lifts the dynamics on the state space to a curve in the transformation group.

Our aim in this paper is to generalize the time-dependent curve in the transformation group to a stochastic process, and then use Hamilton's principle to determine the stochastic dynamics of the momentum map taking values in the dual of the Lie algebra of the transformation group.

Our approach to achieve the transition from deterministic to stochastic geometric mechanics is to concentrate on the Lie algebra action $\dot{q}(t)=-u q(t) \in T Q$ of the vector field $u=g^{-1} \dot{g} \in \mathfrak{g}$, which produces the solution paths $q(t) \in Q$. The solution paths $q(t)=g^{-1}(t) q_{0} \in Q$ will become stochastic, if $g(t)$ is made stochastic by replacing the deterministic reconstruction equation $g^{-1} \dot{g}=u$ mentioned above by introducing the following reconstruction relation from a stochastic vector field,

$$
g^{-1} d_{t} g=u d t+\sum_{i} \xi_{i} \circ d W^{i}(t)
$$

where subscripted $d_{t}$ represents stochastic time evolution, the vector fields $\xi_{i}$ for $i=1,2, \ldots, N$, are prescribed, and $\circ d W^{i}(t)$ denotes the Stratonovich differential with independent Brownian motions $d W^{i}(t)$. The idea, then, is to regard the stochastic solution paths $q(t)=g(t)^{-1} q_{0} \in Q$ as observable data, from which we obtain the correlation eigenvectors $\xi_{i}$ by some form of bespoke data assimilation, and substitute them into the constrained action

$$
\int_{a}^{b}\left(\ell\left(u, g^{-1} q_{0}\right)+\left\langle m, g^{-1} d_{t} g-u d t-\sum_{i} \xi_{i} \circ d W^{i}(t)\right\rangle_{\mathfrak{g}}\right) d t
$$

then take variations to derive the corresponding equations of motion for $u \in \mathfrak{g}$ by applying Poincaré's approach to the resulting stochastically constrained Hamilton's principle. In this way, we obtain a variational approach for deriving data-driven models in the framework of stochastic geometric mechanics.

\section{$1.2 \quad$ Data-driven modeling of uncertainty}

As opposed to theory-driven models such as Newtonian force laws and thermodynamic processes for the subgridscale dynamics, here we will use stochastic geometric mechanics as an opportunity to consider a new type of data-driven modeling. In data-driven modeling, one seeks to model properties of a subsystem of a given dynamical system which, for example, may be observable at length or time scales which are below the resolution of available initial and boundary conditions, or of numerical simulations of the dynamical system based on the assumed exact equations.

The most familiar example of data-driven modeling occurs in numerical weather forecasting, where various numerically unresolvable, but observable, subgrid-scale processes are expected to have profound effects on the variability of the weather; so they must be parameterized at the resolved scales of the numerical simulations. Of 
course, the accuracy of a given parameterization model often remains uncertain. In fact, even the possibility of modeling subgrid-scale properties in terms of resolved-scale quantities simulations may sometimes be questionable. However, if some information about the statistics of the small-scale excitations is known, such as the spatial correlations of its observed transport properties at the resolved scales, one may arguably consider modeling the effects of the small scale dynamics on the resolved scales by a stochastic transport process whose spatial correlations match the observations, at the resolved scales. In this case, the eigenvectors of the correlation matrix of the observations may provide the modes of the sub-scale motion, to be modeled by applying stochasticity with those statistics at the resolved scales. Although fluid dynamics is not considered in the present work, it falls within the purview of geometric mechanics and has been a source of inspiration in the previous development of stochastic geometric mechanics [17].

Stochastic perturbations in finite dimensions. As an example of data-driven modeling in finite dimensions, we consider the following situation. Suppose one notices an erratic "jitter" or "wobble" in the motion of an observable quantity, $q(t)=g(t)^{-1} q_{0}$ whose dynamics is governed by a subsystem of the full dynamics. For example, one might observe a jitter in the angular velocity of an orbiting satellite, indicated, say, by a small antenna attached to it. Being only a subsystem quantity, and satisfying an auxiliary equation implying that it merely follows the rigid motions of the satellite, this observable quantity certainly does not determine the motion of the full system. However, the observation of its motion could still contain some useful information. For example, suppose its statistics can be measured. One may ask what dynamics of the full system would give rise to the observed statistics of the subsystem. In particular, one would be interested to know whether the observation of a perturbative wobble found in a subsystem could mean that the motion of the full system would eventually destabilize. If the dynamics of the unperturbed full system follows from Hamilton's principle, constrained by a deterministic auxiliary equation for an observable quantity, $q(t)$, then a reasonable procedure might be to take the variations, subject to the constraints determined from one's observations of the wobble in the subsystem, described as a stochastic perturbation of the original auxiliary equation for $q(t)$. Equivalently, given the observation of an apparently stochastic perturbation in a subsystem, one might ask, what motion equation gives rise to this stochastic wobble in the subsystem? In general, of course, this is not a well-posed question. However, for the geometric mechanics systems posed here, this question will have a definite answer.

The rigid body example. Euler's equation for stochastic motion for a rigid body provides a useful example in finite dimensions. For the Euler rigid body equations, the stochasticity introduced via the present approach enters the angular velocity and thereby provides a geometric mechanics description of stochastic motion of the angular momentum. In this type of problem, one asks, for example, whether an observed erratic perturbation in the angular velocity may destabilize a deterministic rigid body equilibrium. Indeed, it can, with positive probability. One also asks what the stochasticity does to the evolution of the energy and other conservation laws. Here the answer is interesting and suggestive of other potentially rich results. The first part of the answer is that the rigid body's energy is no longer conserved, but the magnitude of its angular momentum is still conserved, since the dynamics describes stochastic coadjoint motion. The rigid body example and the related heavy top example, when gravity is present, have been treated in [1, 2].

\subsection{Stochastic Hamilton equations}

On the Hamiltonian side, the modern name for Poincaré's "new form" of dynamics is "coadjoint motion". The primary source of stochastic symplectic Hamilton equations is 4, which was recently reviewed and developed further from the geometric mechanics viewpoint in [21. In the present work, we are also interested in the situation where the motion is generated by applying a transformation group to a configuration manifold $Q$ with coordinates $q$, and then extending its action by cotangent lift to its entire phase space $T^{*} Q$ with coordinates $(q, p)$. The primary example occurs when the rotation group $G=S O(3)$ acts on $Q=\mathbb{R}^{3}$ and Poincaré's new form of the motion equation governs the angular momentum $J(q, p) \in \mathfrak{s o}(3)^{*} \simeq \mathbb{R}^{3}$ of the rigid body, or heavy top, or spherical pendulum. This situation requires the noise to be present in both the $q$ and $p$ equations. Bismut's 1981 book [4 discusses the 
Hamiltonian dynamics of stochastic particle motion, in which

$$
d q=\left\{q, H(q, p) d t+\sum_{i} h_{i}(q, p) \circ d W^{i}(t)\right\}, \quad d p=\left\{p, H(q, p) d t+\sum_{i} h_{i}(q, p) \circ d W^{i}(t)\right\},
$$

for the canonical Poisson bracket $\{\cdot, \cdot\}$. If the stochastic Hamiltonians $h_{i}(q)$ happen to depend only on position $q$, then stochasticity appears only in the canonical momentum equation, as a Newtonian force,

$$
d q=\{q, H(q, p) d t\}, \quad d p=\left\{p, H(q, p) d t+\sum_{i} h_{i}(q) \circ d W^{i}(t)\right\}
$$

In this restricted case, the difference between Stratonovich and Itô noise is immaterial. However, for rotating motion in three dimensions $q \in \mathbb{R}^{3}$, for example, we will need the stochastic Hamiltonians $h_{i}(q, p)$ to depend on both $q$ and $p$, since $q$ and $p$ transform the same way under rotations. In particular, they both transform as vectors in $\mathbb{R}^{3}$. In this situation, the noise appears in both of the equations in (1.5), and the difference between Stratonovich and Itô noise is crucial. The distinction between Stratonovich and Itô noise is important for all of the motion equations in Poincaré's form, since the transformation of the conjugate momentum $p$ is the cotangent lift of the transformation of coordinate $q$ in Poincaré's class of equations.

\subsection{A distinction from other approaches}

Although Poincaré 27] used a version of what one would now call "reduction by symmetry", here we use an earlier approach due to Clebsch [9, which introduces constrained variations into Hamilton's principle by imposing velocity maps corresponding in the deterministic case to the infinitesimal transformations of a Lie group. (For up to date applications to mechanics of the Clebsch method, see [13.) In a certain sense, Clebsch 9 presages the Pontryagin maximum principle in optimal control theory. In the present paper, however, the velocity maps will be made stochastic.

Thus, we will consider stochastic Clebsch action principles whose variables are stochastic. The equations of motion derived will be stochastic ordinary (or partial) differential equations (SDEs, or SPDEs) for motion on coadjoint orbits of (finite or infinite dimensional) Lie algebras.

Now we comment further on the distinction between the stochastic Clebsch and reduced Lagrangian approaches. A stochastic Lagrangian symmetry reduction process has been developed in [3, 8]. In that case, the Lagrangian curves in the configuration space are stochastic diffusion processes, which are critical states of the action functional. In these works, the drift of the stochastic processes is essentially regarded as its (mean, generalized) time derivative. In [3, the action functional is defined with the classical Lagrangian computed on that velocity; the corresponding Euler-Poincaré equations of motion, satisfied by the velocity, are deterministic (ordinary differential equations when the configuration space is finite-dimensional, or partial differential equations in the infinite-dimensional case). In [8] the same kind of reduction process for stochastically perturbed Lagrangians is considered and corresponding stochastic differential equations of motion (stochastic partial differential equations in the infinite dimensional case) are derived.

In the present paper, as in [17, the stochastic Clebsch approach is not equivalent to the reduced stochastic Lagrangian processes approach employed in [3, 8. In particular, the velocities in the reduction approach of [3, 8] are essentially identified with the drift of the underlying diffusion processes, which, as is well known, requires the computation of a conditional expectation. In addition, in the reduced stochastic Lagrangian approach of [3, 8], it is not possible to take arbitrary variations; instead, a particular form for the variations is required and the final resulting equations of motion depend on this choice. However, in the present work and in [17, the variations are quite arbitrary.

Therefore, the present stochastic Clebsch action principle cannot be regarded as a formulation of the EulerPoincaré variational principle obtained in [8]. In order to consider the present variational principle approach from the viewpoint of reduction by symmetry, one would need to interpret the velocity as an Itô derivative of the underlying stochastic curves, in which case the resulting stochastic action functional would be divergent. This divergence was avoided in [3, 8, via the "renormalization" achieved by taking conditional expectations. 
Outline of the paper. Following the Clebsch approach to the Euler-Poincaré equations, in Section2 we introduce a stochastic velocity map in the Stratonovich sense as a constraint in Hamilton's principle for motion on a manifold acted upon by infinitesimal transformations of a Lie algebra. With hindsight, we see that the stochasticity in the velocity map is coupled to the motion by the momentum map which arises from the variation of the Lagrangian function and the deterministic part of the velocity map. The resulting stationarity conditions generalize the classical deterministic formulations of motion on coadjoint orbits of Lie algebras in Poincaré [27] and Hamel [15, by making them stochastic. In Section 3, we present the Itô formulations of the stationary variational conditions. Three alternative routes are taken in calculating the Itô double-bracket forms of the variational equations for stochastic coadjoint motion. In Section 4 , we discuss the Poisson structure of the Stratonovich-Hamiltonian formulation of the stochastic motion equations. We also give the Itô interpretation of the Casimir functions for the Lie-Poisson part of the bracket in this formulation, and derive the associated Lie-Poisson Fokker-Planck equation for the motion of the probability density function on the level sets of Casimir functions.

\section{Variational principle for Stratonovich stochastic coadjoint motion}

\subsection{Deterministic formulation}

In 27], Poincaré begins by considering the transitive action of a Lie group $G$ of smooth transformations of a manifold $Q$, whose points in local coordinates are written as $q=\left(q^{1}, \ldots, q^{n}\right)$ and whose infinitesimal transformations are represented by the vector field obtained at linear order in the Taylor series. Let $\left\{e_{1}, \ldots, e_{r}\right\}$ be a basis of $\mathfrak{g}$ and $\alpha=1, \ldots, r$ the indices of the local coordinates in this basis. Denote by $A_{\alpha}[f]$ any infinitesimal transformation of this group, and express its action on a smooth function $f$ as

$$
A_{\alpha}[f]:=\sum_{i=1}^{n} A_{\alpha}^{i} \frac{\partial f}{\partial q^{i}}=A_{\alpha}^{1} \frac{\partial f}{\partial q^{1}}+A_{\alpha}^{2} \frac{\partial f}{\partial q^{2}}+\cdots+A_{\alpha}^{n} \frac{\partial f}{\partial q^{n}}, \quad \alpha=1, \ldots, r,
$$

where $A_{\alpha}^{i}$ are functions of $\left(q^{1}, \ldots, q^{n}\right)$.

Throughout this paper, Greek indices enumerate Lie algebra basis elements, Latin indices denote coordinates on the manifold, and the standard Einstein summation convention is assumed. Since these transformations form a Lie algebra, Poincaré remarks that

$$
A_{\alpha}\left[A_{\beta}\right]-A_{\beta}\left[A_{\alpha}\right]=\sum_{\gamma=1}^{r} c_{\alpha \beta}^{\gamma} A_{\gamma} \Longleftrightarrow A_{\alpha}^{s} \frac{\partial A_{\beta}^{k}}{\partial q^{s}}-A_{\beta}^{s} \frac{\partial A_{\alpha}^{k}}{\partial q^{s}}=c_{\alpha \beta}^{\gamma}(q) A_{\gamma}^{k}, \quad \forall k=1, \ldots, n, \alpha, \beta=1, \ldots, r,
$$

where $c_{\alpha \beta}{ }^{\gamma}(q) \in C^{\infty}(Q)$ are structure functions for the Lie algebra of smooth vector fields on the manifold $Q$, in the basis associated with the Greek indices. When the $A_{\alpha}^{i}(q)$ are linear functions of $\left(q^{1}, \ldots, q^{n}\right)$, then the $c_{\alpha \beta}{ }^{\gamma} \in \mathbb{R}$ are the usual structure constants of a matrix Lie algebra. In this regard, Poincaré [27] presages Hamel [15], cf. also Marle [23.

Geometric setup. We give now a glimpse of the global formulation. Poincaré [27] does not really use a transformation group, only its associated Lie algebra action. In [27. Poincaré takes a configuration $n$-manifold $Q$ of a mechanical system and a Lie algebra morphism $\mathfrak{g} \ni u \mapsto u_{Q} \in \mathfrak{X}(Q)$ of a given Lie algebra $\mathfrak{g}$, dim $\mathfrak{g}=: r<\infty$, to the Lie algebra $\mathfrak{X}(Q)$ of vector fields on $Q$, endowed with the usual Lie bracket $[X, Y][f]:=X[Y[f]]-Y[X[f]]$, where $X, Y \in \mathfrak{X}(Q), f \in C^{\infty}(Q)$, and $X[f]$ is the differential of $f$ in the direction $X$, given in coordinates by (2.1). The coordinate expression

$$
u_{Q}(q)=: u_{Q}^{i}(q) \frac{\partial}{\partial q^{i}}=: A_{\alpha}^{i}(q) u^{\alpha} \frac{\partial}{\partial q^{i}}
$$

of $u_{Q} \in \mathfrak{X}(Q)$, relative to a coordinate system $\left(q^{1}, \ldots, q^{n}\right)$ on the chart domain $U \subset Q$ and a basis $\left\{e_{1}, \ldots, e_{r}\right\}$ of $\mathfrak{g}$, is thus determined by the functions $A_{\alpha}^{i} \in C^{\infty}(U)$ and the basis expansion $u=: u^{\alpha} e_{\alpha}$ of $u \in \mathfrak{g}$. Since $\left[u_{Q}, v_{Q}\right]=[u, v]_{Q}$ for any $u, v \in \mathfrak{g}$, the functions $A_{\alpha}:=\left[A_{\alpha}^{i}\right] \in C^{\infty}\left(U, \mathbb{R}^{n}\right)$, defined by $\left(e_{\alpha}\right)_{Q}=: A_{\alpha}^{i} \frac{\partial}{\partial q^{i}}$, satisfy (2.2) ${ }^{1}$, which is

\footnotetext{
${ }^{1}$ Thus, Poincaré works with a right action of the underlying Lie group on the manifold; we adopt his index conventions in [27, also used in [7]. For left actions, $\mathfrak{g} \rightarrow \mathfrak{X}(Q)$ is a Lie algebra anti-homomorphism, i.e., $\left[u_{Q}, v_{Q}\right]=-[u, v]_{Q}$.
} 
equivalent to saying that the local vector fields $A_{\alpha}, A_{\beta} \in \mathfrak{X}(U)$ satisfy

$$
\left[A_{\alpha}, A_{\beta}\right] \stackrel{[2.2]}{=} c_{\alpha \beta}{ }^{\gamma}(q) A_{\gamma}
$$

The action is assumed to be transitive in 27, which means that any tangent vector $v_{q} \in T_{q} Q$ is of the form $v_{q}=u_{Q}(q)$ for some $u \in \mathfrak{g}$, and hence if $u=a^{\alpha} e_{\alpha}$ for some $a^{\alpha} \in \mathbb{R}$, then $v_{q}$ can be written locally as $v_{q}=$ $a^{\alpha}\left(e_{\alpha}\right)_{Q}(q)=a^{\alpha} A_{\alpha}^{i}(q) \frac{\partial}{\partial q^{i}}$.

If $\left(q^{1}, \ldots, q^{n}\right)$ are local coordinates on $Q$, the corresponding standard coordinates on the tangent bundle $T Q$ and the cotangent bundle $T^{*} Q$ are, respectively, $\left(q^{1}, \ldots, q^{n}, \dot{q}^{1}, \ldots, \dot{q}^{n}\right)$ and $\left(q^{1}, \ldots, q^{n}, p_{1}, \ldots, p_{n}\right)$, where $v_{q}=\dot{q}^{i} \frac{\partial}{\partial q^{i}}$ and $p_{q}=p_{i} d q^{i}$ for any $v_{q} \in T_{q} Q$ and $p_{q} \in T_{q}^{*} Q$ (the cotangent space at $q \in Q$, the dual of $T_{q} Q$ ). Throughout the paper, we use these naturally induced coordinates. The sign convention for the canonical Poisson bracket on $T^{*} Q$ adopted in this paper is, in standard coordinates,

$$
\{f, g\}=\frac{\partial f}{\partial q^{k}} \frac{\partial g}{\partial p_{k}}-\frac{\partial g}{\partial q^{k}} \frac{\partial f}{\partial p_{k}}, \text { for any } f, g \in C^{\infty}\left(T^{*} Q\right) .
$$

If $h \in C^{\infty}\left(T^{*} Q\right)$, its Hamiltonian vector field is denoted by $X_{h}^{T^{*} Q} \in \mathfrak{X}\left(T^{*} Q\right)$. Hamilton's equations for a curve $c(t) \in T^{*} Q$ in Poisson bracket form are $\frac{d}{d t} f(c(t))=\{f, h\}(c(t))$ for any $f \in C^{\infty}\left(T^{*} Q\right)$.

When working with a general Poisson manifold $(P,\{\cdot, \cdot\})$, the Hamiltonian vector field $X_{h}^{P} \in \mathfrak{X}(P)$ of $h \in C^{\infty}(P)$ is defined by $\mathbf{d} f\left(X_{h}^{P}\right):=\{f, h\}$. For a symplectic manifold, $(P, \omega)$, this is equivalent to the usual definition, $\left.\mathbf{i}_{X_{h}^{P}} \omega=X_{h}^{P}\right\lrcorner \omega=\mathbf{d} h$.

Pairing notation. For any manifold $Q$, finite or infinite dimensional, we denote by $\langle\cdot, \cdot\rangle_{Q}: T^{*} Q \times T Q \rightarrow \mathbb{R}$ the natural (weakly, in the infinite-dimensional case) non-degenerate fiberwise duality pairing. Given a Lie algebra $\mathfrak{g}$, which is always finite dimensional in this paper, the non-degenerate duality pairing between its dual $\mathfrak{g}^{*}$ and $\mathfrak{g}$ is denoted by $\langle\cdot, \cdot\rangle_{\mathfrak{g}}: \mathfrak{g}^{*} \times \mathfrak{g} \rightarrow \mathbb{R}$.

Given $f \in C^{\infty}\left(\mathfrak{g}^{*}\right)$, the functional derivative $\frac{\delta f}{\delta \mu} \in \mathfrak{g}$ of $f$ evaluated at $\mu \in \mathfrak{g}^{*}$ is defined by

$$
\left.\frac{d}{d \epsilon}\right|_{\epsilon=0} f(\mu+\epsilon \delta \mu)=\left\langle\delta \mu, \frac{\delta f}{\delta \mu}\right\rangle_{\mathfrak{g}}, \quad \text { for all } \delta \mu \in \mathfrak{g} .
$$

The momentum map. The momentum map $\mathbf{J}_{T^{*} Q}: T^{*} Q \rightarrow \mathfrak{g}^{*}$ of the lifted $\mathfrak{g}$-action to $T^{*} Q$ is defined by

$$
u_{T^{*} Q}=X_{\mathbf{J}^{u}}^{T^{*} Q}, \quad \text { for any } u \in \mathfrak{g},
$$

where $\mathbf{J}_{T^{*} Q}^{u}\left(p_{q}\right):=\left\langle\mathbf{J}_{T^{*} Q}\left(p_{q}\right), u\right\rangle_{\mathfrak{g}}$. Its expression is, cf. $\S 12.1$, formula (12.1.15) of [24],

$$
\mathbf{J}_{T^{*} Q}^{u}\left(p_{q}\right)=\left\langle p_{q}, u_{Q}(q)\right\rangle_{Q}, \quad p_{q} \in T^{*} Q, u \in \mathfrak{g}, \quad \text { or, in coordinates, } \quad \mathbf{J}_{T^{*} Q}\left(q^{i}, p_{i}\right)=p_{j} A_{\alpha}^{j}\left(q^{i}\right) e^{\alpha},
$$

where $\left\{e^{1}, \ldots, e^{r}\right\}$ is the basis of $\mathfrak{g}^{*}$ dual to the basis $\left\{e_{1}, \ldots, e_{r}\right\}$ of $\mathfrak{g}$. This momentum map is infinitesimally equivariant. That is 2

$$
\mathbf{J}_{T^{*} Q}^{[u, v]}=-\left\{\mathbf{J}_{T^{*} Q}^{u}, \mathbf{J}_{T^{*} Q}^{v}\right\},
$$

for all $u, v \in \mathfrak{g}$. A useful equivalent statement of infinitesimal equivariance is (see, e.g., [24, §11.5, formula (11.5.6)] with a sign change because we work with right actions)

$$
T_{p_{q}} \mathbf{J}_{T^{*} Q}\left(u_{T^{*} Q}\left(p_{q}\right)\right)=\operatorname{ad}_{u}^{*} \mathbf{J}_{T^{*} Q}\left(p_{q}\right) .
$$

If we denote

$$
m:=\mathbf{J}_{T^{*} Q} \in C^{\infty}\left(T^{*} Q, \mathfrak{g}^{*}\right), \quad m_{\alpha}:=\mathbf{J}_{T^{*} Q}^{e_{\alpha}}, \quad m_{[\alpha, \beta]}:=\mathbf{J}_{T^{*} Q}^{\left[e_{\alpha}, e_{\beta}\right]} \in C^{\infty}\left(T^{*} Q\right),
$$

we have $m=m_{\alpha} e^{\alpha}$, with

$$
m_{\alpha}\left(p_{q}\right)=p_{i} A_{\alpha}^{i}(q)
$$

\footnotetext{
${ }^{2}$ This is the infinitesimal equivariance relation for right actions. For left actions, the sign in the right hand side changes.
} 
and the infinitesimal equivariance is expressed in coordinates as

$$
-m_{[\alpha, \beta]}=\left\{m_{\alpha}, m_{\beta}\right\} \stackrel{\sqrt[(2.5)]{=}}{=}-p_{j}\left[A_{\alpha}, A_{\beta}\right]^{j \stackrel{(2.4)}{=}}-p_{j} c_{\alpha \beta} A_{\gamma}^{j} \stackrel{(2.11)}{=}-c_{\alpha \beta} m_{\gamma} .
$$

If $G$ is a Lie group with Lie algebra $\mathfrak{g}$ acting on the right on $Q$, then the $\mathfrak{g}$-action on $Q$ is given by the infinitesimal generator vector field $u_{Q} \in \mathfrak{X}(Q)$ defined at every $q \in Q$ by $u_{Q}(q):=\left.\frac{d}{d t}\right|_{t=0} q \cdot \exp (t u) \in T_{q} Q$, where $q \cdot g$ denotes the action of $g \in G$ on the point $q \in Q$. The momentum map $\mathbf{J}: T^{*} Q \rightarrow \mathfrak{g}^{*}$ given in (2.8) is equivariant relative to the given right $G$-action on $P$ and the right coadjoint $G$-action on $\mathfrak{g}^{*}$, i.e., $\mathbf{J}_{T^{*} Q}\left(p_{q} \cdot g\right)=\operatorname{Ad}_{g}^{*} \mathbf{J}_{T^{*} Q}\left(p_{q}\right)$ for all $g \in G$. We use here the following notations: $\operatorname{Ad}_{g}: \mathfrak{g} \rightarrow \mathfrak{g}$ is the adjoint action of $g \in G$, defined as the derivative at the identity of the conjugation by $g$ in $G ; \operatorname{Ad}_{g}$ is a Lie algebra isomorphism; $\operatorname{Ad}_{g}^{*}: \mathfrak{g}^{*} \rightarrow \mathfrak{g}^{*}$ is the dual map of $\operatorname{Ad}_{g}$.

The Lie-Poisson bracket. The dual $\mathfrak{g}^{*}$ of any finite dimensional Lie algebra $\mathfrak{g}$ is endowed with the Lie-Poisson bracket (see, e.g., [24, §13.1, p.416])

$$
\{f, h\}_{ \pm}(\mu)= \pm\left\langle\mu,\left[\frac{\delta f}{\delta \mu}, \frac{\delta h}{\delta \mu}\right]\right\rangle_{\mathfrak{g}}, \quad f, h \in C^{\infty}\left(\mathfrak{g}^{*}\right), \quad \mu \in \mathfrak{g}^{*}
$$

We denote by $\mathfrak{g}_{ \pm}^{*}$ the vector space $\mathfrak{g}^{*}$ endowed with the Poisson bracket (2.13). The Hamiltonian vector field of $h \in C^{\infty}\left(\mathfrak{g}^{*}\right)$ defined by the equation $\dot{f}=\{f, h\}$ for any $f \in C^{\infty}\left(\mathfrak{g}^{*}\right)$ has the expression $X_{h}^{ \pm}(\mu)=\mp \operatorname{ad}_{\frac{\delta h}{\delta \mu}}^{*} \mu$ (the signs correspond). Given $\xi \in \mathfrak{g}$, ad $\mathrm{d}_{\xi}^{*}: \mathfrak{g}^{*} \rightarrow \mathfrak{g}^{*}$ is the dual of the linear map $\mathfrak{g} \ni \eta \mapsto \operatorname{ad}_{\xi} \eta:=[\xi, \eta] \in \mathfrak{g}$.

If $G$ is a Lie group with Lie algebra $\mathfrak{g}$, left translation by $g \in G$ is denoted as $G \ni h \mapsto L_{g}(h):=g h \in G$ and, likewise, $G \ni h \mapsto R_{g}(h):=h g \in G$ denotes right translation. Then the momentum map $\mathbf{J}_{R}: T^{*} G \rightarrow \mathfrak{g}_{-}^{*}$, $\mathbf{J}_{R}\left(\alpha_{g}\right)=T_{e}^{*} L_{g}\left(\alpha_{g}\right), \alpha_{g} \in T_{g}^{*} G$, of the lifted right translation on $G$ (i.e., $\mathbf{J}_{R}$ equals $\mathbf{J}_{T^{*} G}$ given in (2.8) for the action of $G$ on itself given by right translations) is a Poisson map, i.e., $\{f, h\}_{-} \circ \mathbf{J}_{R}=\left\{f \circ \mathbf{J}_{R}, h \circ \mathbf{J}_{R}\right\}$. Similarly, the momentum map $\mathbf{J}_{L}: T^{*} G \rightarrow \mathfrak{g}_{+}^{*}, \mathbf{J}_{L}\left(\alpha_{g}\right)=T_{e}^{*} R_{g}\left(\alpha_{g}\right), \alpha_{g} \in T_{g}^{*} G$, for left translation is another Poisson map, i.e., $\{f, h\}_{+} \circ \mathbf{J}_{L}=\left\{f \circ \mathbf{J}_{L}, h \circ \mathbf{J}_{L}\right\}$. (For the proof see, e.g., [24, §13.3].) More generally, the momentum map $\mathbf{J}: T^{*} Q \rightarrow \mathfrak{g}_{-}^{*}$ of the lifted right $\mathfrak{g}$-action to $T^{*} Q$ is a Poisson map; the coordinate expression of this statement is (2.12).

A function $k \in C^{\infty}\left(\mathfrak{g}^{*}\right)$ such that $\{k, f\}_{ \pm}=0$, for all $f \in C^{\infty}\left(\mathfrak{g}^{*}\right)$, or, equivalently, $X_{k}^{ \pm}=0$, is called a Casimir function. This definition is valid for any Poisson manifold, not just $\mathfrak{g}^{*}$.

\subsection{Stochastic Clebsch formulation}

Introducing stochasticity into the Clebsch methodology. We assume that all stochastic processes are defined in the same filtered probability space $\left(\Omega, \mathbb{P}, \mathcal{P}_{t}\right)$. Let $t \mapsto W_{t}^{k}(\omega), k=1, \ldots N, \omega \in \Omega$, be $N$ independent real-valued Brownian motions, $\xi_{1}, \ldots, \xi_{N} \in \mathfrak{g}$, and $\Omega \ni \omega \mapsto\left(p_{q}\right)_{\omega}(t) \in T^{*} Q$ random variables for every $t$. The induced random variable on $Q$, the foot point of $\left(p_{q}\right)_{\omega}(t)$, is denoted by $\Omega \ni \omega \mapsto q_{\omega}(t) \in Q$. Stratonovich differentiation is denoted by $X \circ d Y$ and Itô differentiation simply by $X d Y$. Then, given $\xi_{1}, \ldots, \xi_{N} \in \mathfrak{g}$ and a $\mathfrak{g}$-valued random curve $u(t)$,

$$
t \longmapsto\left\langle\left(p_{q}\right)_{\omega}(t), \circ d q_{\omega}(t)-u_{\omega}(t)_{Q}\left(q_{\omega}(t)\right) d t-\left(\xi_{k}\right)_{Q}\left(q_{\omega}(t)\right) \circ d W_{t}^{k}(\omega)\right\rangle_{Q}
$$

is a process whose coordinate expression is

$$
\left(p_{i}\right)_{\omega}(t)\left(\circ d q_{\omega}^{i}(t)-A_{\alpha}^{i}\left(q_{\omega}(t)\right) u_{\omega}^{\alpha}(t) d t-A_{\alpha}^{i}\left(q_{\omega}(t)\right) \xi_{k}^{\alpha} \circ d W_{t}^{k}\right) .
$$

We always assume that the stochastic processes are defined for all times $t \in[0, T]$, the coefficients are smooth, and that $u(t)$ is smooth in the time variable. Furthermore, we assume that the manifold $Q$ has no boundary.

Remark on notation. For simplicity in the notation, we no longer write the probability variable $\omega$ and, instead, we use symbols $p, q$, etc., to denote semimartingales. 
Given the Lagrangian $\ell \in C^{\infty}(\mathfrak{g} \times Q)$, introduce the stochastic action, defined for random curves $u \in C^{1}([0, T], \mathfrak{g})$, $q \in C([0, T], Q),\left(p_{q}\right) \in C\left([0, T], T^{*} Q\right)$, and define the constrained stochastic action integral $S\left(u, p_{q}\right)$ by

$$
S\left(u, p_{q}\right)=\int_{0}^{T} \ell(u(t), q(t)) d t+\left\langle p_{q}(t), \circ d q(t)-u(t)_{Q}(q(t)) d t-\left(\xi_{k}\right)_{Q}(q(t)) \circ d W_{t}^{k}\right\rangle,
$$

where the semimartingale $p_{q}$ is assumed to be regular enough for the above integrals to be finite. Indeed, all stochastic processes considered in this paper will be continuous semimartingales with regular coefficients. In local coordinates, the stochastic action integral (2.14) may be recognized as the sum of a Lebesgue integral and a Stratonovich integral

$$
S\left(u, p_{q}\right)=\underbrace{\int_{0}^{T}\left(\ell(u(t), q(t)) d t-p_{i}(t) A_{\alpha}^{i}(q(t)) u^{\alpha}(t) d t\right)}_{\text {Lebesgue integral }}+\underbrace{\int_{0}^{T} p_{i}\left(\circ d q^{i}(t)-A_{\alpha}^{i}(q(t)) \xi_{k}^{\alpha} \circ d W_{t}^{k}\right)}_{\text {Stratonovich integral }} .
$$

For notational convenience, we introduce for every $t \in[0, T]$ the stochastic Lie algebra element whose components in the basis $\left\{e_{1}, \ldots, e_{r}\right\}$ of $\mathfrak{g}$ are

$$
d x_{t}^{\alpha}:=u^{\alpha}(t) d t+\xi_{k}^{\alpha} \circ d W_{t}^{k},
$$

to convey that, when we integrate some stochastic process $X_{t}$ with respect to $d x_{t}^{\alpha}$, we mean

$$
\int_{0}^{T} X_{t} d x_{t}^{\alpha}:=\int_{0}^{T} X_{t} u^{\alpha}(t) d t+\int_{0}^{T} X_{t} \xi_{k}^{\alpha} \circ d W_{t}^{k}, \quad \alpha=1, \ldots, \operatorname{dim} \mathfrak{g}=r .
$$

In particular, we rewrite the action integral in (2.15) in the abbreviated form

$$
S\left(u, p_{q}\right)=\int_{0}^{T} \ell(u(t), q(t)) d t+p_{i}(t)\left(\circ d q^{i}(t)-A_{\alpha}^{i}(q(t)) d x_{t}^{\alpha}\right) .
$$

We assume that the Lagrangian $\ell(u, q)$, viewed as a function $\ell: \mathfrak{g} \times Q \rightarrow \mathbb{R}$, is hyperregular, i.e., for every $q \in Q$, the map $\mathfrak{g} \times\{q\} \ni(u, q) \mapsto n:=\frac{\delta \ell}{\delta u} \in \mathfrak{g}^{*} \times\{q\}$ is a diffeomorphism. In particular, $n$ is a function of $(u, q)$ and, conversely, $u$ is a function of $(n, q)$. Thus, replacing the variables $u \in \mathfrak{g}$ and $q \in Q$ by the random curves $u(t), q(t)$, we get the semimartingale $n(u(t), q(t))$.

Consider a random point $\left(q_{\omega}, p_{\omega}\right)$ in the manifold $T^{*} Q$ and $f \in C^{\infty}\left(T^{*} Q\right)$. The differential of $f$ in the direction of the (deterministic) vector field $Z \in \mathfrak{X}\left(T^{*} Q\right)$ is given by

$$
\langle\mathbf{d} f, Z\rangle_{Q}\left(q_{\omega}, p_{\omega}\right)=\left.\frac{d}{d \epsilon}\right|_{\epsilon=0} f\left(\gamma_{\omega}(\epsilon)\right)
$$

where $\gamma_{\omega}$ is a curve starting from $\left(q_{\omega}, p_{\omega}\right)$ with initial velocity $Z\left(q_{\omega}, p_{\omega}\right)$ and the limit is taken in $L^{2}(\Omega)$. Therefore $\langle\mathbf{d} f, Z\rangle_{Q}\left(q_{\omega}, p_{\omega}\right)$ consists in evaluating $\langle\mathbf{d} f, Z\rangle_{Q}$ at the random point $\left(q_{\omega}, p_{\omega}\right)$.

Consider now a semimartingale of the form

$$
\mathscr{Y}_{t}(q, p)=\mathscr{Y}_{0}+\int_{0}^{t} \phi_{\alpha}(q(s), p(s)) \xi_{k}^{\alpha} \circ d W_{s}^{k}+\int_{0}^{t} \psi(q(s), p(s)) d s
$$

where $q(t), p(t)$ are $Q$-, respectively, $T^{*} Q$-valued semimartingales, with $q(t)$ the footpoint of $p(t), \phi_{\alpha}, \psi \in$ $C^{\infty}\left(T^{*} Q\right)$ are deterministic smooth functions, and $\xi_{k}=\xi_{k}^{\alpha} e_{\alpha} \in \mathfrak{g}$ are given (constant) elements. The (Stratonovich) stochastic Poisson bracket of $f(q(t), p(t))$ with $\mathscr{Y}_{t}$ is defined by

$$
\begin{aligned}
\left\{f(q(t), p(t)), \circ d_{t} y_{t}\right\}: & =\left\langle\mathbf{d} f, X_{\phi_{\alpha}}\right\rangle_{Q}(q(t), p(t)) \xi_{k}^{\alpha} \circ d W_{t}^{k}+\left\langle\mathbf{d} f, X_{\psi}\right\rangle_{Q}(q(t), p(t)) d t \\
& =\left\{f, \phi_{\alpha}\right\}(q(t), p(t)) \xi_{k}^{\alpha} \circ d W_{t}^{k}+\{f, \psi\}(q(t), p(t)) d t .
\end{aligned}
$$

where $X_{\phi}, X_{\psi}$ denote the Hamiltonian vector fields of $\phi$ and $\psi$. 
If $g \in C^{\infty}\left(T^{*} Q\right)$, the Poisson bracket of the two semimartingales $f(q(t), p(t))$ and $g(q(t), p(t))$ is defined as

$$
\{f(q(t), p(t)), g(q(t), p(t))\}:=\{f, g\}(q(t), p(t)),
$$

i.e., it equals the semimartingale obtained by computing the function $\{f, g\} \in C^{\infty}\left(T^{*} Q\right)$ and replacing its variables $(q, p)$ by the semimartingales $(q(t), p(t))$.

The constraint imposed by the pairing with the Lagrange multipliers $p_{i}(t)$ defines the $i$ th component of the stochastic velocity map,

$$
d q^{i}(t)=A_{\alpha}^{i}(q(t)) d x_{t}^{\alpha} .
$$

To justify the computations that follow on manifolds and ensure that they are intrinsic, we provide a quick review of the basics of the Malliavin Calculus in the next subsection.

\subsection{Calculus of variations on path spaces}

In this subsection we give a brief summary of some definitions and results about the calculus of variations on (probability) path spaces known as Malliavin Calculus, both in the case where the paths take values on Euclidean spaces and on Riemannian manifolds. For this subject we refer to [22].

Malliavin derivative: the Euclidean case. Beginning with the Euclidean configuration space case, let $x_{0} \in \mathbb{R}^{n}$ be given, and fixed throughout the discussion below, and let $\mathbb{P}_{x_{0}}=\left\{x:[0, T] \rightarrow \mathbb{R}^{n}, x\right.$ continuous, $\left.x(0)=x_{0}\right\}$ be the path space of continuous paths endowed with the law $\mu$ of the Brownian motion on $\mathbb{R}^{n}$ starting from $x_{0}$ at time 0 and with the usual past filtration $\mathcal{P}_{t}$. A variation of the paths $x$ is a map $z:[0, T] \rightarrow \mathbb{R}^{n}$ of bounded variation and such that $\int_{0}^{T}\left|\frac{d}{d t} z(t)\right|^{2} d t<\infty, z(0)=0$. These are the elements in the Cameron-Martin space, which is dense in $\mathbb{P}_{x_{0}}$ for the sup topology. For a functional $F \in L_{\mu}^{p}\left(\mathbb{P}_{x_{0}}\right)$, the Malliavin derivative of $F$ in the direction $z$ is defined as

$$
D_{z} F(x)=\lim _{\epsilon \rightarrow 0} \frac{1}{\epsilon}(F(x+\epsilon z)-F(x)),
$$

the limit being taken in the $L_{\mu}^{2}$ sense. For a "cylindrical" functional of the form $F(x)=f(x(t))$, for each $t \in[0, T]$ fixed, where $f$ is a real valued smooth (at least $C^{1}$ ) function, we have

$$
D_{z} f(x(t))=\frac{\partial f}{\partial x^{i}}(x(t)) z^{i}(t) .
$$

For a semimartingale $\zeta$ with values in $\mathbb{R}^{n}$ we can also define $D_{\zeta} f(x(t))=\frac{\partial f}{\partial x^{i}}(x(t)) \zeta^{i}(t)$.

If we consider pinned Brownian paths (or bridges), the corresponding path space will be $\mathbb{P}_{x_{0}, x_{T}}=\{x:[0, T] \rightarrow$ $\mathbb{R}^{n}, x$ continuous, $\left.x(0)=x_{0}, x(T)=x_{T}\right\}$. In this case the variations must satisfy the condition $z(T)=0$.

The Itô map. Analogously, given a smooth $n$-dimensional manifold $Q$ acted upon by a Lie group $G$ with Lie algebra $\mathfrak{g}$, and a point $q_{0} \in Q$, let $\mathbb{P}_{q_{0}}(Q):=\left\{q:[0, T] \rightarrow Q \mid q\right.$ continuous, $\left.q(0)=q_{0}\right\}$ denote the path space of continuous paths starting at $q_{0}$, endowed with the law of the process

$$
d q(t):=\left(\xi_{k}\right)_{Q}(q(t)) \circ d W_{t}^{k}+(u(t))_{Q}(q(t)) d t,
$$

with $q(0)=q_{0}$. As usual, $\eta_{Q}$ denotes the infinitesimal generator vector field on $Q$ induced by $\eta \in \mathfrak{g}, \xi_{1}, \ldots, \xi_{N} \in \mathfrak{g}$ are $N$ given Lie algebra elements, and $t \mapsto u(t) \in \mathfrak{g}$ is a given random path that we assume adapted and of bounded variation (we are actually assuming smoothness). In addition, we request that the process $d q(t)$ defined above does not explode in finite time and, in particular, is defined for all $t \in[0, T]$. form

It is possible to define in the space $\mathbb{P}_{q_{0}}(Q)$ a global chart, as follows. On the manifold $Q$ we consider the bilinear

$$
\bar{g}(q)\left(p_{q}, \beta_{q}\right):=\sum_{k=1}^{N} p_{q}\left(\left(\xi_{k}\right)_{Q}(q)\right) \beta_{q}\left(\left(\xi_{k}\right)_{Q}(q)\right), \quad p_{q}, \beta_{q} \in T_{q}^{*} Q
$$


whose coordinate expression is

$$
\bar{g}^{i j}=\sum_{k=1}^{N} A_{\alpha}^{i} \xi_{k}^{\alpha} A_{\beta}^{j} \xi_{k}^{\beta}=\sum_{k=1}^{N}\left(\xi_{k}\right)_{Q}^{i}\left(\xi_{k}\right)_{Q}^{j}
$$

It is assumed that this is a co-metric on $T^{*} Q$, i.e., $\bar{g}$ is positive definite, so that the diffusions $q$ are non singular, i.e., their generators are elliptic. The associated Riemannian metric on $Q$, denoted by $g$ or $\langle\langle\cdot, \cdot\rangle\rangle$, has a corresponding Levi-Civita connection $\nabla$. A (stochastic) parallel transport over the paths $q(\cdot)$ can be defined (following Itô, see for example, [19]). We denote it by $t_{\tau_{2} \leftarrow \tau_{1}}^{q}: T_{q\left(\tau_{1}\right)} Q \rightarrow T_{q\left(\tau_{2}\right)} Q$.

This amounts to solving the following stochastic system

$$
d_{\tau_{1}}\left[t_{\tau_{2} \leftarrow \tau_{1}}^{q}\right]_{i}^{j}=\left[t_{\tau_{2} \leftarrow \tau_{1}}^{q}\right]_{r}^{j} \Gamma_{s i}^{r}\left(A_{\alpha}^{s}(q(t)) \xi_{k}^{\alpha} \circ d W_{t}^{k}+A_{\alpha}^{s}(q(t)) u^{\alpha} d t\right), \quad\left[t_{\tau_{2} \leftarrow \tau_{2}}^{q}\right]=I,
$$

where $\Gamma$ are the Christoffel symbols associated with the Levi-Civita connection $\nabla, i, j, r, s=1, \ldots, n$, and $k=$ $1, \ldots, N$. Parallel transport of vector fields can be lifted to parallel transport of orthonormal frames, namely isometries $r: \mathbb{R}^{n} \rightarrow T_{q} Q$ (c.f. [19]). For a path $W \in \mathbb{P}_{x_{0}}$, let $r_{W}(t)$ be the parallel transport of frames from time 0 to time $t$ along $W(t)$, with $\pi\left(r_{W}(0)\right)=q_{0}$ (where $\pi$ denotes the canonical projection from the orthonormal frame bundle over $Q$ to the underlying manifold $Q$ ). We have $t_{\tau_{2} \leftarrow \tau_{1}}^{q}=r_{W}\left(\tau_{2}\right) r_{W}\left(\tau_{1}\right)^{-1}$.

The Itô map $\mathcal{I}: \mathbb{P}_{x_{0}}\left(\mathbb{R}^{n}\right) \rightarrow \mathbb{P}_{q_{0}}(Q)$, is defined by

$$
\mathcal{I}(W)(t)=\pi\left(r_{W}(t)\right)
$$

This map realizes an isomorphism of probability spaces, i.e., it is a bijective map that transports the law of the Brownian motion to the law of the process $q(22])$. It provides a global chart for the path space of the manifold that will be used throughout the paper.

Malliavin derivative: the manifold case. A variation of the path $q \in \mathbb{P}_{q_{0}}(Q)$ is a map $Z_{q}(t) \in T_{q(t)} Q$ such that $z(t)=t_{0 \leftarrow t}^{q}\left(Z_{q}(t)\right)$ is a variation on the Euclidean path space $\mathbb{P}_{x_{0}}\left(\mathbb{R}^{n}\right)$ as defined above. We have

$$
\frac{d}{d t} z(t)=t_{0 \leftarrow t}^{q}\left(\nabla_{t}^{q} Z(t)\right),
$$

where $\nabla^{q}$ is the covariant derivation $\nabla_{t}^{q} Z_{q}(t)=\lim _{\epsilon \rightarrow 0} \frac{1}{\epsilon}\left(t_{t \leftarrow t+\epsilon}^{q}\left(Z_{q}(t+\epsilon)\right)-Z_{q}(t)\right)$.

Then, for a cylindrical functional $F$ defined on the path space $\mathbb{P}_{q_{0}}(Q)$ of the form $F(q)=f(q(t))$, for each $t \in[0, T]$ fixed, we consider its directional derivative

$$
D_{Z} F(q)=\int_{0}^{T} 1_{\tau<t}\left(t_{0 \leftarrow t}^{q}(\nabla f)(q(t))\right)^{i} \frac{d}{d \tau} z_{i}(\tau) d \tau,
$$

where $1_{\tau<t}$ denotes the characteristic function of the open interval $(-\infty, t)$.

These Malliavin derivatives can be defined for more general functionals, but in this paper we only need those which are introduced above. Notice that for $Q=\mathbb{R}^{n}$, we have $D_{z} F(x)=\frac{\partial f}{\partial x^{i}}(x(t)) z_{i}(t)$. That is, the directional derivative coincides with the one defined in the Euclidean path space setting.

The pull back of the Malliavin derivative to Euclidean space. We want to pull back derivatives on the path space of the manifold to the Euclidean path space. For this purpose, we invoke the following result (22, ch. $\mathrm{XI}]$, or [5, ch. II c] and [10] for the case with drift):

$$
\left(D_{Z} F\right) \circ \mathcal{I}=D_{\zeta}(F \circ \mathcal{I})
$$

where the $\mathbb{R}^{n}$-valued semimartingale $\zeta$ satisfies the stochastic differential system

$$
\left\{\begin{array}{c}
d \zeta(t)=\frac{d}{d t} z(t) d t-\left(\frac{1}{2} R_{t}+\mathcal{D}(t)\right)(z(t)) d t-\rho(t) d W(t) \\
d \rho(t)=\Omega(\circ d W(t)-b(t) d t, z(t))
\end{array}\right.
$$


Here, $z(t):=t_{0 \leftarrow t}^{q}\left(Z_{q}(t)\right)$ for the given the path $t \mapsto Z_{q}(t) \in T_{q(t)} Q, \Omega: T Q \times T Q \rightarrow T Q$ is the curvature tensor of the metric $g$ on $Q$,

$$
R_{t}=t_{0 \leftarrow t}^{q} \circ \operatorname{Ricci}_{q(t)} \circ t_{t \leftarrow 0}^{q}
$$

is the representation in the global chart of the Ricci tensor on $Q$, and $\mathcal{D}(t)=t_{0 \leftarrow t}^{q} \circ \nabla b(t) \circ t_{t \leftarrow 0}^{q}$, where $b(t)$ is the time dependent smooth vector field on $Q$ defined by

$$
b(t):=(u(t))_{Q}+\frac{1}{2} \sum_{k=1}^{N} \nabla_{\left(\xi_{k}\right)_{Q}}\left(\xi_{k}\right)_{Q} .
$$

Considering the variation $Z$ on the path space of the manifold corresponds to considering in the global chart given by the Itô map a variation with respect to the semimartingale $\zeta$ defined above. The diffusion part of this semimartingale is given by the curvature of the manifold, which is antisymmetric; therefore this part corresponds to a rotation of the Brownian motion, which is again a Brownian motion. We neglect this rotation and understand that we may not be working with a fixed Brownian motion, but eventually with equivalent ones (identical in law). Therefore, our variations with respect to $Z$ will be taken, using the global chart, with respect to directions $\bar{z}$ of the form

$$
\frac{d}{d t} \bar{z}=\frac{d}{d t} z(t)-\left(\frac{1}{2} R+\mathcal{D}\right)(z(t)) .
$$

The map $z \mapsto \bar{z}$ can be inverted, through the resolvent equation

$$
\frac{d}{d t} Q_{t, s}=\left(\frac{1}{2} R+\mathcal{D}\right) Q_{t, s}, \quad Q_{s, s}=I d
$$

and $z(t)=\int_{0}^{t} Q_{t, s}\left(\frac{d}{d s} \bar{z}(s)\right) d s$, as long as the previous resolvent equation has a solution for all $t \in[0, T]$.

We can then conclude that making an "arbitrary" variation in the direction $Z$ in the path space of the manifold corresponds, using the global chart, to making an "arbitrary" variation in $\mathbb{P}_{x_{0}}\left(\mathbb{R}^{n}\right)$ in the direction $z$ (an adapted random curve of bounded variation with time derivative in $L_{\mu}^{2}$ ). To use a more intuitive notation we shall denote by $\delta q(t)=Z_{q}(t)$ a variation in $\mathbb{P}_{q_{0}}(Q)$.

All the considerations above still hold for pinned Brownian paths, namely those with a final condition $q(T)=q_{T}$. Then the variations are equal to zero at this final time. The corresponding sigma-algebra and filtration on the path space are the usual ones, generated by the coordinate maps and generated by the coordinate maps up to time $t$, respectively. We refer to [11, 12] for more explanation.

From now on, we also assume a growth control on the vector fields $u(t)$ so that the laws of the corresponding diffusion processes $d q(t):=\left(\xi_{k}\right)_{Q}(q(t)) \circ d W_{t}^{k}+(u(t))_{Q}(q(t)) d t, q(0)=q_{0}$ are absolutely continuous with respect to the law of $d \tilde{q}(t):=\left(\xi_{k}\right)_{Q}(\tilde{q}(t)) \circ d W_{t}^{k}, \tilde{q}(0)=\tilde{q}_{0}$. The standard assumption to ensure this is Novikov's condition (see, e.g., [19); namely, that there exists a $\mathbb{R}^{N}$-valued stochastic process $\theta(t)$ such that $\left(\xi_{k}\right)_{Q} \theta^{k}(t)=u(t)_{Q}$ and a constant $0<\lambda<1$ such that $E_{\mu}\left(\exp \lambda \int_{0}^{T}|\theta(t)|^{2} d t\right)<\infty$. Also we assume growth control on $u$ and $b$ so that the resolvent equation above has a solution defined for all $t \in[0, T]$.

\subsection{Stochastic variational principles}

With these definitions and preparatory formulas, the following theorem holds.

Theorem 1. [Stratonovich stochastic variational principle] The stochastic variational principle $\delta S=0$ with action integral $S$ given in equation (2.17), with $d x_{t}^{\alpha}$ given by (2.16), defined on diffusion processes with fixed diffusion coefficients, under the assumptions described before and such that $q(0)=q_{0}, q(T)=q_{T}$, yields the following stochastic dynamical equations

$$
\begin{aligned}
& d_{t} n_{\alpha}(q(t), p(t))=\left\{n_{\alpha}(q(t), p(t)), n_{\beta}(q(t), p(t))\right\} d x_{t}^{\beta}+\frac{\partial \ell}{\partial q^{i}}(u(t), q(t)) A_{\alpha}^{i}(q(t)) d t, \\
& d_{t} q^{i}(t)=\left\{q^{i}(t), n_{\beta}(q(t), p(t))\right\} d x_{t}^{\beta}, \quad d_{t} p_{i}(t)=\left\{p_{i}(t), n_{\beta}(q(t), p(t))\right\} d x_{t}^{\beta}+\frac{\partial \ell}{\partial q^{i}}(u(t), q(t)) d t,
\end{aligned}
$$


for all $\alpha=1, \ldots, \operatorname{dim} \mathfrak{g}, i=1, \ldots, \operatorname{dim} Q$, where $n:=\frac{\delta \ell}{\delta u} \in \mathfrak{g}^{*}$, i.e., $n_{\alpha}=\frac{\partial \ell}{\partial u^{\alpha}}$. Moreover, $n(q(t), p(t))=$ $m(q(t), p(t))$ a.s., where $m\left(p_{q}\right)=\mathbf{J}_{T^{*} Q}\left(p_{q}\right)=m_{\alpha}\left(p_{q}\right) e^{\alpha} \in \mathfrak{g}^{*}, m_{\alpha}=p_{i} A_{\alpha}^{i}(q)$ and hence $m_{\alpha}(q, p)=p_{i} A_{\alpha}^{i}(q)$.

The Poisson brackets in this formula need interpretation, since $n_{\alpha}$ depends only on the variables $u$ and $q^{i}$. First, the Poisson brackets on the right hand side of (2.23) are taken in the sense of (2.20) or its global version. Second, as will be shown below, the stationarity condition $\delta S=0$ yields the relation $\frac{\delta \ell}{\delta u^{\alpha}}(q(t), p(t))=p_{i}(t) A_{\alpha}^{i}(q(t))$ almost surely, which says that $n(q(t), p(t))=m(q(t), p(t))$ almost surely and that the Lagrange multipliers $p_{i}(t)$ also depend on the random curves $u(t)$ and $q^{i}(t)$, as expected. By pretending now that the quantity $m_{\alpha}=p_{i} A_{\alpha}^{i}(q)$ depends on the variables $q^{i}$ and $p_{i}$, as if they were independent $T^{*} Q$-chart variables, one may compute the Poisson brackets in (2.23) by using the derivative of the semimartingale $\{f, g\}(q(t), p(t))$.

Remark 2. Upon looking back at the Stratonovich integral in the stochastic action functional (2.15), we see that the stochasticity couples to the phase space variables through the momentum map via the relations $\frac{\partial \ell}{\partial u^{\alpha}}(u(t), q(t))=$ $p_{i}(t) A_{\alpha}^{i}(q(t))$.

Proof. The first step in the proof of Theorem 1 is to take the variations of the action integral (2.15), thereby finding the following equations, which hold almost surely,

$$
\begin{array}{ll}
\delta u(t): & \frac{\delta \ell}{\delta u^{\alpha}}(u(t), q(t))-p_{i}(t) A_{\alpha}^{i}(q(t))=0, \\
\delta p(t): & d_{t} q^{i}(t)-A_{\alpha}^{i}(q(t)) d x_{t}^{\alpha}=0, \\
\delta q(t): & d_{t} p_{i}(t)+p_{j}(t) \frac{\partial A_{\alpha}^{j}}{\partial q^{i}}(q(t)) d x_{t}^{\alpha}-\frac{\partial \ell}{\partial q^{i}}(u(t), q(t)) d t=0,
\end{array}
$$

after integrations by parts using the vanishing of the term $\left.\left(p_{i}(t) \delta q^{i}(t)\right)\right|_{0} ^{T}$ at the endpoints in time, which follows from the assumption $\delta q^{i}(0)=0=\delta q^{i}(T)$.

Notice that we are not fixing a priori a vector field $u(t)$ in this Theorem. Nevertheless, by Novikov's condition above, the laws of the corresponding diffusions are absolutely continuous with respect a fixed path space (strictly before the final time $T$ ) and all the admissible variations, as we have seen, do not depend on the form of $u(t)$. Admissible variations for the stochastic process $q(\cdot)$, in the global chart given by the Ito map, are variations on the corresponding path space as defined in (2.22). Therefore one can take arbitrary (in this sense) horizontal variations of the process $p(\cdot)_{q(\cdot)}$. Since also variations in all vertical directions are allowed, we can take arbitrary variations of $p(\cdot)_{q(\cdot)}$.

On the contrary, the admissible paths $(q$ and $p$ ) for which the action functional $S$ is defined have fixed diffusion coefficients, so that we can use Malliavin calculus on the corresponding path spaces. Since the diffusion coefficient of $p$ is not elliptic at every point, we may regularize it, deduce the corresponding dynamical equations and then pass to the limit.

In particular, we have $n_{\alpha}(q(t), p(t))=\frac{\delta \ell}{\delta u^{\alpha}}(u(t), q(t))=m_{\alpha}(q(t), p(t))$. Therefore, taking the stochastic differential of the first equation, then using the second and third equations in (2.24), we get, dropping the $t$ - 
dependence notation in the semimartingales,

$$
\begin{aligned}
d_{t} m_{\alpha}(q, p) & =d\left(\frac{\delta \ell}{\delta u^{\alpha}}\right)=d\left(p_{i} A_{\alpha}^{i}(q)\right)=\left(\circ d p_{i}\right) A_{\alpha}^{i}(q)+p_{i} \frac{\partial A_{\alpha}^{i}}{\partial q^{j}}(q) \circ d q^{j} \\
& \stackrel{(2.16)}{=} A_{\alpha}^{i}(q)\left(-p_{j} \frac{\partial A_{\beta}^{j}}{\partial q^{i}}(q) d x_{t}^{\beta}+\frac{\partial \ell}{\partial q^{i}}(u, q) d t\right)+p_{j} \frac{\partial A_{\alpha}^{j}}{\partial q^{k}}(q) A_{\beta}^{k}(q) d x_{t}^{\beta} \\
& =R_{j}\left(A_{\beta}^{k}(q) \frac{\partial A_{\alpha}^{j}}{\partial q^{k}}(q)-A_{\alpha}^{k}(q) \frac{\partial A_{\beta}^{j}}{\partial q^{k}}(q)\right) d x_{t}^{\beta}+\frac{\partial \ell}{\partial q^{i}}(u, q) A_{\alpha}^{i}(q) d t \\
& \stackrel{(2.2)}{=} R_{j} c_{\beta \alpha^{\gamma}}{ }^{\gamma} A_{i} A_{\gamma}^{j}(q) d x_{t}^{\beta}+\frac{\partial \ell}{\partial q^{i}} A_{\alpha}^{i}(q) d t \\
& =-c_{\alpha \beta} m_{\gamma}(q, p) d x_{t}^{\beta}+\frac{\partial \ell}{\partial q^{i}}(u, q) A_{\alpha}^{i}(q) d t \\
& \stackrel{(2.12)}{=}\left\{m_{\alpha}(q, p), m_{\beta}(q, p)\right\} d x_{t}^{\beta}+\frac{\partial \ell}{\partial q^{i}}(u, q) A_{\alpha}^{i}(q) d t
\end{aligned}
$$

which is the first equation in (2.23).

The second equation $d_{t} q^{i}=A_{\alpha}^{i}(q) d x_{t}^{\alpha}$ in (2.24) and the identity $\left\{q^{i}, m_{\beta}(q, p)\right\}=A_{\beta}^{i}(q)$ yield the second equation in (2.23). Finally, the third equation $d_{t} p_{i}=-p_{j} \frac{\partial A_{\alpha}^{j}}{\partial q^{i}}(q) d x_{t}^{\alpha}+\frac{\partial \ell}{\partial q^{i}}(u, q) d t$ in (2.24) and the identity $\left\{p_{i}, m_{\beta}(q, p)\right\}=-p_{j} \frac{\partial A_{\beta}^{j}}{\partial q^{i}}(q)$ yield the third equation in (2.23).

The first variational equation in (2.24) captures the momentum map relation (2.11), and the latter two equations in (2.24) produce the corresponding equations in (2.23), when expressed in terms of the canonical Poisson bracket $\{\cdot, \cdot\}$ on $T^{*} Q$. The second equation in (2.24) recovers the velocity map in (2.17), and the third equation determines the evolution of the dual canonical momentum variable, the Lagrange multiplier $p_{i}$.

The penultimate equality in (2.25) yields the following result.

Corollary 3. Hamilton's principle $\delta S=0$ for the constrained action integral in (2.17) recovers stochastic coadjoint motion equation in the following form,

$$
d_{t}\left(\frac{\partial \ell}{\partial u^{\alpha}}(u(t), q(t))\right)=\left(\operatorname{ad}_{d x_{t}}^{*} \frac{\partial \ell}{\partial u}(u(t), q(t))\right)_{\alpha}+\frac{\partial \ell}{\partial q^{i}}(u(t), q(t)) A_{\alpha}^{i}(q(t)) d t,
$$

where $\left(\operatorname{ad}_{d x_{t}}^{*} \frac{\partial \ell}{\partial u}(u(t), q(t))\right)_{\alpha}:=-c_{\alpha \beta} \gamma \frac{\partial \ell}{\partial u^{\gamma}}(u(t), q(t)) d x_{t}^{\beta}$.

\subsection{The stochastic equations of motion on $\mathfrak{g}^{*} \times Q$}

The presence of the Poisson brackets in (2.23) suggests the existence of a Hamiltonian version of these equations. This will be explored in detail in Section 4 . Here we just introduce a stochastic version of the Legendre transform and derive certain equations on $\mathfrak{g}^{*} \times Q$ whose geometric structure will be investigated in Section 4

In the classical deterministic case, recall that the Legendre transform of a Lagrangian $\mathfrak{L}: \mathfrak{g} \rightarrow \mathbb{R}$ to a Hamiltonian $\mathfrak{H}: \mathfrak{g}^{*} \rightarrow \mathbb{R}$, mapping the Euler-Poincaré equations $\frac{d}{d t} \frac{\delta \mathfrak{L}}{\delta \xi}=-\operatorname{ad}_{\xi}^{*} \frac{\delta \mathfrak{L}}{\delta \xi}$ to the Lie-Poisson equations $\frac{d}{d t} \mu=-\operatorname{ad}_{\frac{\delta, 5}{\delta \mu}}^{*} \mu$ (and, conversely, if the map is a diffeomorphism), is given by (see, e.g., [24, §13.5, p. 437])

$$
\mu:=\frac{\delta \mathfrak{L}}{\delta \xi}, \quad \mathfrak{H}(\mu):=\langle\mu, \xi\rangle_{\mathfrak{g}}-\mathfrak{L}(\xi), \quad \xi \in \mathfrak{g}, \quad \mu \in \mathfrak{g}^{*} .
$$

If the map $\mathfrak{g} \ni \xi \mapsto \mu=\frac{\delta \mathfrak{L}}{\delta \xi} \in \mathfrak{g}^{*}$ is a diffeomorphism, the Lagrangian $\mathfrak{L}$ and Hamiltonian $\mathfrak{H}$ given above, are called hyperregular. We define below a stochastic version of this Legendre transform, depending on a parameter, replacing the Lie algebra element by the stochastic vector field (2.16) and the element in the dual of the Lie algebra by a semimartingale. 
We proceed in the following way. We say that the Lagrangian $\ell: \mathfrak{g} \times Q \rightarrow \mathbb{R}$ is hyperregular if the function $\ell(\cdot, q): \mathfrak{g} \rightarrow \mathbb{R}$ is hyperregular for every $q \in Q$. We work with hyperregular Lagrangians from now on. Define, as in Theorem $10:=n(u, q):=\frac{\delta \ell}{\delta u} \in \mathfrak{g}^{*}$, invert this relation for every $q \in Q$ to get $u=u(n, q)$, and introduce the Hamiltonian function $h(n, q):=\langle n, u(n, q)\rangle_{\mathfrak{g}}-\ell(u(n, q), q)$. Next, recalling that $m=\mathbf{J}_{T^{*} Q}\left(p_{q}\right)=p_{i} A_{\alpha}^{i}(q) e^{\alpha} \in \mathfrak{g}^{*}$, consider the function $h(m, q)$, i.e., we replace the first variable $n$ of $h$ by the expression $m$. Now, replace the variables $(u, q) \in \mathfrak{g} \times Q$ by random curves $(u(t), q(t))$ and form a semimartingale $h(m(q(t), p(t)), q(t))$ (which corresponds to a stochastic Hamiltonian, as explained in subsection 4.2), by imposing, in analogy with the deterministic case, the stochastic derivative of the semimartingale $h(m(q(t), p(t)), q(t))$ to equal

$$
d_{t} h(m(q(t), p(t)), q(t))=p_{i}(t) A_{\alpha}^{i}(q(t)) d x_{t}^{\alpha}-\ell(u(t), q(t)) d t \stackrel{2.11}{=} m_{\alpha}(q(t), p(t)) d x_{t}^{\alpha}-\ell(u(t), q(t)) d t
$$

where $d x_{t}^{\alpha}=u^{\alpha} d t+\xi_{k}^{\alpha} \circ d W_{t}^{k}$.

This semimartingale is of the form (2.18), namely

$$
d h(m(q(t), p(t)), q(t))=\left(h^{1}\right)_{\alpha}(m(q(t), p(t)), q(t)) \xi_{k}^{\alpha} \circ d W_{t}^{k}+h^{2}(m(q(t), p(t)), q(t)) d t
$$

In agreement with our previous definitions, we shall use the notation

$$
\begin{gathered}
d_{t}\left(\frac{\partial h}{\partial m_{\beta}}\right)(m(q(t), p(t)), q(t))=\frac{\partial\left(h^{1}\right)_{\alpha}}{\partial m_{\beta}}(m(q(t), p(t)), q(t)) \xi_{k}^{\alpha} \circ d W_{t}^{k}+\frac{\partial h^{2}}{\partial m_{\alpha}}(m(q(t), p(t)), q(t)) d t, \\
d_{t}\left(\frac{\partial h}{\partial q^{j}}\right)(m(q(t), p(t)), q(t))=\frac{\partial\left(h^{1}\right)_{\alpha}}{\partial q^{j}}(m(q(t), p(t)), q(t)) \xi_{k}^{\alpha} \circ d W_{t}^{k}+\frac{\partial h^{2}}{\partial q^{j}}(m(q(t), p(t)), q(t)) d t .
\end{gathered}
$$

Theorem 4. The stochastic variational principle $\delta S=0$, with action integral defined in (2.17) and semimartingale $h(m(q(t), p(t)), q(t))$ introduced above, implies the equations

$$
\begin{aligned}
& d_{t} m_{\alpha}(q(t), p(t))=\left\{m_{\alpha}(q(t), p(t)), m_{\beta}(q(t), p(t))\right\} d x_{t}^{\beta}-A_{\alpha}^{j}(q(t)) \frac{\partial h}{\partial q^{j}}(m(q(t), p(t)), q(t)) d t \\
&=\{\left.m_{\alpha}(q(t), p(t)), m_{\beta}(q(t), p(t))\right\} \circ d_{t}\left(\frac{\partial h}{\partial m_{\beta}}\right)(m(q(t), p(t)), q(t)) \\
& \quad+\left\{m_{\alpha}(q(t), p(t)), q^{j}\right\} \frac{\partial h}{\partial q^{j}}(m(q(t), p(t)), q(t)) d t, \\
& d_{t} q^{i}(t)=\left\{q^{i}, m_{\beta}(q(t), p(t))\right\} d x_{t}^{\beta}=A_{\beta}^{i}(q(t)) d x_{t}^{\beta}=A_{\beta}^{i}(q(t)) \circ d_{t}\left(\frac{\partial h}{\partial m_{\beta}}\right)(m(q(t), p(t)), q(t)),
\end{aligned}
$$

with the convention that the Poisson brackets are computed as in Theorem 1.

Proof. In the computations below, we shall drop the notational $t$-dependence of the semimartingales. By Theorem 1. we know that $n(q, p)=m(q, p)$ a.s. and that (2.23) hold. Next, we take the differential of condition (2.27). Thus, if $\delta m_{\alpha}(q, p)$ and $\delta q^{i}$ are arbitrary variations (namely random curves of bounded variation in $t$ ) of the semimartingales $m_{\alpha}(q, p)$ and $q^{i}$, respectively, we get

$$
\begin{aligned}
& d\left(\frac{\partial h}{\partial m_{\alpha}}\right)(m(q, p), q) \delta m_{\alpha}(q, p)+d\left(\frac{\partial h}{\partial q^{i}}\right)(m(q, p), q) \delta q^{i} \\
& =\delta m_{\alpha}(m(q, p)) d x_{t}^{\alpha}+\delta u^{\alpha}\left(m_{\alpha}(m(q, p))-\frac{\partial \ell}{\partial u^{\alpha}}(u, q)\right) d t-\delta q^{i}\left(\frac{\partial \ell}{\partial q^{i}}\right)(u, q) d t
\end{aligned}
$$

which is equivalent a.s. to

$$
\begin{aligned}
& m_{\alpha}(q, p)-\frac{\partial \ell}{\partial u^{\alpha}}(u, q)=0, \quad d\left(\frac{\partial h}{\partial m_{\alpha}}\right)(m(q, p), q)=d x_{t}^{\alpha}, \quad \text { and } \\
& d\left(\frac{\partial h}{\partial q^{i}}\right)(m(q, p), q)=-\left(\frac{\partial \ell}{\partial q^{i}}\right)(u, q) d t .
\end{aligned}
$$


Note that the first equation implies, as expected from Theorem 1 the a.s. equality of the semimartingales $n(q, p)=$ $m(q, p)$.

Using the identities (2.31) and the equations (2.23), we compute $d m_{\alpha}(q, p)$ and $d q^{i}$ to find,

$$
\begin{aligned}
d m_{\alpha}(q, p) & =\left\{m_{\alpha}(q, p), m_{\beta}(q, p)\right\} d x_{t}^{\beta}-A_{\alpha}^{j}(q) \frac{\partial h}{\partial q^{j}}((q, p), q) d t \\
& \stackrel{2.31}{=}\left\{m_{\alpha}(q, p), m_{\beta}(q, p)\right\} \circ d\left(\frac{\partial h}{\partial m_{\beta}}((q, p), q)\right)+\left\{m_{\alpha}(q, p), q^{j}\right\} \frac{\partial h}{\partial q^{j}}((q, p), q) d t, \\
d q^{i} & =\left\{q^{i}, m_{\beta}(q, p)\right\} d x_{t}^{\beta}=A_{\beta}^{i}(q) d x_{t}^{\beta} \stackrel{2.31}{=} A_{\beta}^{i}(q) \circ d\left(\frac{\partial h}{\partial m_{\beta}}\right)((q, p), q),
\end{aligned}
$$

which recover equations (2.30).

Remark 5. The defining relation $m_{\alpha}(q, p)=p_{i} A_{\alpha}^{i}(q)$ and the second equation in (2.23) imply

$$
\begin{gathered}
d m_{\alpha}(q, p)=d\left(p_{i} A_{\alpha}^{i}(q)\right)=\left(\circ d p_{i}\right) A_{\alpha}^{i}(q)+p_{i} \frac{\partial A_{\alpha}^{i}}{\partial q^{j}}(q) \circ d q^{j} \\
\stackrel{2.23]}{=}\left(\circ d p_{i}\right) A_{\alpha}^{i}(q)+p_{i} \frac{\partial A_{\alpha}^{i}}{\partial q^{j}}(q) A_{\beta}^{j}(q) d x_{t}^{\beta} .
\end{gathered}
$$

By Theorem [ we know that $n(q, p)=m(q, p)$ a.s. and hence the first equation in (2.23) yields

$$
\begin{aligned}
d m_{\alpha}(q, p) & =\left\{m_{\alpha}(q, p), m_{\beta}(q, p)\right\} d x_{t}^{\beta}+\frac{\partial \ell}{\partial q^{i}}(u, q) A_{\alpha}^{i}(q) d t \\
\stackrel{2.25}{=} & R_{j}\left(A_{\beta}^{k}(q) \frac{\partial A_{\alpha}^{j}}{\partial q^{k}}(q)-A_{\alpha}^{k}(q) \frac{\partial A_{\beta}^{j}}{\partial q^{k}}(q)\right) d x_{t}^{\beta}+\frac{\partial \ell}{\partial q^{i}}(u, q) A_{\alpha}^{i}(q) d t .
\end{aligned}
$$

Comparing these two expressions, we conclude the a.s. equality

$$
\left(\circ d p_{i}\right) A_{\alpha}^{i}(q)=-p_{j} A_{\alpha}^{k}(q) \frac{\partial A_{\beta}^{j}}{\partial q^{k}}(q) d x_{t}^{\beta}+\frac{\partial \ell}{\partial q^{i}}(u, q) A_{\alpha}^{i}(q) d t=\left(-p_{j} \frac{\partial A_{\beta}^{j}}{\partial q^{i}}(q) d x_{t}^{\beta}+\frac{\partial \ell}{\partial q^{i}}(u, q) d t\right) A_{\alpha}^{i}(q) .
$$

Note that this identity is clearly implied by the third equation in (2.23).

\section{Itô formulation of stochastic coadjoint motion}

As before, $t \mapsto W_{t}^{k}(\omega), k=1, \ldots N, \omega \in \Omega$, are $N$ independent real-valued Brownian motions and $\xi_{1}, \ldots, \xi_{N} \in \mathfrak{g}$. For each $\xi_{k}, k=1, \ldots, N$, define the Hamiltonian vector field $X_{\xi_{k}} \in \mathfrak{X}\left(T^{*} Q\right)$ by

$$
X_{\xi_{k}}:=\left\{\cdot, m_{\alpha}(q, p) \xi_{k}^{\alpha}\right\} \stackrel{2.24}{=}\left\{\cdot, p_{i} A_{\alpha}^{i}(q) \xi_{k}^{\alpha}\right\},
$$

i.e., $X_{\xi_{k}}$ is the Hamiltonian vector field on $T^{*} Q$ with Hamiltonian function $T^{*} Q \ni p_{q} \mapsto\left\langle\mathbf{J}_{T^{*} Q}\left(p_{q}\right), \xi_{k}\right\rangle_{\mathfrak{g}} \in \mathbb{R}$, $k=1, \ldots, N$. As in the previous section, we denote interchangeably points in $T^{*} Q$ by $p_{q}$ or $(q, p)$.

Define the operator on semimartingales of the form $f(q(t), p(t))$, where $f \in C^{\infty}\left(T^{*} Q\right)$ by

$$
\left(X_{\xi_{k}} f\right)(q(t), p(t)):=\left\{f(q(t), p(t)), m_{\alpha}(q(t), p(t)) \xi_{k}^{\alpha}\right\}=\left\{f(q(t), p(t)), p_{i}(t) A_{\alpha}^{i}(q(t)) \xi_{k}^{i}\right\},
$$

where the brackets in the right hand side are those of semimartingales, as in (2.20). Note that the result of the operation $\left(X_{\xi_{k}} f\right)(q(t), p(t))$, defined in (3.2), is again a semimartingale.

In analogy with (2.16), define the Itô stochastic element $d \widehat{x}_{t}^{\beta} \in \mathfrak{g}$ by

$$
d \widehat{x}_{t}^{\alpha}:=u^{\alpha}(t) d t+\xi_{k}^{\alpha} d W_{t}^{k} .
$$


The Itô stochastic Hamiltonian vector field $\mathscr{X}_{d \widehat{x}_{t}}$ is also defined by the Poisson bracket operation

$$
\left(x_{d \widehat{x}_{t}} f\right)(q(t), p(t)):=\left\{f(q(t), p(t)), m_{\beta}(q(t), p(t))\right\} d \widehat{x}_{t}^{\beta}:=\left\{f(q(t), p(t)), p_{i}(t) A_{\beta}^{i}(q(t))\right\} d \widehat{x}_{t}^{\beta},
$$

for any $f \in C^{\infty}\left(T^{*} Q\right)$, where, again, the brackets in the right hand side are those of semimartingales, (2.20). The result of the operation $\left(X_{d \widehat{x}_{t}} f\right)(q(t), p(t))$, defined in (3.4), is again a semimartingale. In both (3.2) and (3.4), in agreement with the conventions in Section 2 we define the semimartingales $q^{i}(t):=q^{i}(q(t), p(t))$ and $p_{i}(t):=p_{i}\left(q(t), p_{(t))}\right.$.

With these notations, we have the following result.

Corollary 6. [Itô stochastic variational conditions] The corresponding Itô forms of the Stratonovich stochastic variational equations (2.23) are given by

$$
\begin{aligned}
d_{t} m_{\alpha}(q(t), p(t)) & =\left(X_{d \widehat{x}_{t}} m_{\alpha}\right)(q(t), p(t))+\frac{1}{2} \sum_{k=1}^{N}\left(X_{\xi_{k}}\left(X_{\xi_{k}} m_{\alpha}\right)\right)(q(t), p(t)) d t+\frac{\partial \ell}{\partial q^{i}}(u(t), q(t)) A_{\alpha}^{i}(q(t)) d t \\
d_{t} q^{i}(t) & =X_{d \widehat{x}_{t}} q^{i}(t)+\frac{1}{2} \sum_{k=1}^{N} X_{\xi_{k}}\left(X_{\xi_{k}} q^{i}\right)(t) d t \\
d_{t} p_{i}(t) & =X_{d \widehat{x}_{t}} p_{i}(t)+\frac{1}{2} \sum_{k=1}^{N} X_{\xi_{k}}\left(X_{\xi_{k}} p_{i}\right)(t) d t+\frac{\partial \ell}{\partial q^{i}}(u(t), q(t)) d t
\end{aligned}
$$

Remark 7. Remarkably, the Itô interpretation for the coadjoint dynamics of the momentum map defined by $m_{\alpha}(q(t), p(t)):=\frac{\partial \ell}{\partial u^{\alpha}}(u(t), q(t))=p_{i}(t) A_{\alpha}^{i}(q(t))$ has the same double bracket structure as the individual equations for the phase space variable $(q, p)$. Several perspectives of how this preservation of structure in Corollary 6 occurs, can be seen by considering three different direct proofs of it.

First proof. In all the proofs below, we ignore the $t$-dependence notation on the semimartingales. The first proof of Corollary 6 begins by streamlining the notation in the Stratonovich stochastic equations (2.23) of Theorem 11, to write them simply as

$$
d m_{\alpha}(q, p)=\left(X_{d x_{t}} m_{\alpha}\right)(q, p)+\frac{\partial \ell}{\partial q^{i}}(u, q) A_{\alpha}^{i}(q) d t, \quad d q^{i}=X_{d x_{t}} q^{i}, \quad d p_{i}=X_{d x_{t}} p_{i}+\frac{\partial \ell}{\partial q^{i}}(u, q) d t,
$$

in terms of the following Poisson bracket operator (analogous to (3.4))

$$
x_{d x_{t}}:=\left\{\cdot, m_{\beta}(q, p)\right\} d x_{t}^{\beta}:=\left\{\cdot, p_{i} A_{\beta}^{i}(q)\right\} d x_{t}^{\beta} .
$$

We want to write these expressions in Itô form. For this, we recall Itô's formula: if $X_{t}$ is a semimartingale with regular coefficients and $f$ a smooth function (c.f., for example, [19]), then

$$
d_{t} f\left(X_{t}\right)=\partial_{i} f\left(X_{t}\right) \circ d_{t} X_{t}^{i}=\partial_{i} f\left(X_{t}\right) d_{t} X_{t}^{i}+\frac{1}{2} \partial_{i, j}^{2} f\left(X_{t}\right) d_{t} X_{t}^{i} \cdot d_{t} X_{t}^{j}
$$

The corresponding Itô forms of the latter Stratonovich expressions in (2.23) are then written equivalently as

$$
\begin{aligned}
& d q^{i}=X_{d \widehat{x}_{t}} q^{i}+\frac{1}{2} \sum_{k=1}^{N} X_{\xi_{k}}\left(X_{\xi_{k}} q^{i}\right) d t \\
& d p_{i}=x_{d \widehat{x}_{t} p_{i}}+\frac{1}{2} \sum_{k=1}^{N} X_{\xi_{k}}\left(X_{\xi_{k} p_{i}}\right) d t+\frac{\partial \ell}{\partial q^{i}}(u, q) d t .
\end{aligned}
$$

We prove the first relation in (3.11), as the other one is similarly derived. To simplify notation, we write simply $A_{\alpha}^{i}$ instead of $A_{\alpha}^{i}(q)$. Recall that

$$
d q^{i}=A_{\alpha}^{i} u^{\alpha} d t+A_{\alpha}^{i} \xi_{k}^{\alpha} \circ d W_{t}^{k}
$$


By Itô's formula (3.10), the only term which is not of bounded variation in the expression for $d A_{\alpha}^{i}$ is equal to $\frac{\partial}{\partial q^{j}}\left(A_{\alpha}^{i}\right) A_{\beta}^{j} \xi_{k}^{\beta} d W_{t}^{k}$ and we conclude that

$$
d\left(A_{\alpha}^{i} \xi_{k}^{\alpha} \xi_{k}^{\alpha}\right) \cdot d W_{t}^{k}=\sum_{k=1}^{N} \frac{\partial A_{\alpha}^{i}}{\partial q^{j}} A_{\beta}^{j} \xi_{k}^{\beta} \xi_{k}^{\alpha} d t .
$$

Thus, (3.10) yields

$$
d q^{i}=A_{\alpha}^{i} u^{\alpha} d t+\left(A_{\alpha}^{i} \xi_{k}^{\alpha}\right) d W_{t}^{k}+\frac{1}{2} \sum_{k=1}^{N} \frac{\partial A_{\alpha}^{i}}{\partial q^{j}} A_{\beta}^{j} \xi_{k}^{\beta} \xi_{k}^{\alpha} d t,
$$

which is the expanded version of the first equation in (3.11).

Having introduced this streamlined notation for $d q^{i}$ and $d p_{i}$ in the Itô equations (3.11), we calculate the Itô equation for the components of the momentum map $m_{\alpha}(q, p):=p_{i} A_{\alpha}^{i}(q)$, by using the Itô rule for the derivative of a product of a pair of Itô semimartingales, $\mathcal{X}$ and $\mathcal{Y}$, given by

$$
d(x y)=x d y+\mathscr{Y} d x+d x \cdot d \mathscr{Y}, \quad \text { and for } d x=\sigma d W, \quad d \mathscr{Y}=\tilde{\sigma} d W \quad \text { we have } \quad d x \cdot d \mathscr{Y}=\sigma \tilde{\sigma} d t,
$$

where $d \mathscr{X} . d \mathscr{Y}$ is the co-variation, or Itô contraction. According to the Itô product rule, the Itô contraction in computing $d m_{\alpha}(q, p)=d\left(p_{i} A_{\alpha}^{i}(q)\right)$ from equation (3.11) is

$$
d p_{i} . d A_{\alpha}^{i}=\sum_{k=1}^{N}\left(X_{\xi_{k}} p_{i}\right) \cdot\left(X_{\xi_{k}} A_{\alpha}^{i}(q)\right) .
$$

Indeed, this Itô contraction expression comes from the fact that the martingale parts of the processes $p_{i}$ and $A_{\alpha}^{i}(q)$ are given, respectively, by

$$
d R_{i} \simeq-k_{j} \frac{\partial A_{\alpha}^{j}}{\partial q^{i}}(q) \xi_{k}^{\alpha} d W_{t}^{k} \quad \text { and } \quad d A_{\alpha}^{i}(q) \simeq \frac{\partial A_{\alpha}^{i}}{\partial q^{j}}(q) A_{\beta}^{j}(q) \xi_{k}^{\beta} d W_{t}^{k}
$$

where $\Phi \simeq \Psi$ means that $\Phi-\Psi$ is a process of bounded variation.

Remarkably, this Itô contraction (3.13) turns out to be exactly what we need to show by direct calculation from (3.11) that

$$
\begin{aligned}
d m_{\alpha}(q, p)= & \left(X_{d \widehat{x}_{t}} m_{\alpha}\right)(q, p)+\frac{1}{2} \sum_{k=1}^{N}\left(X_{\xi_{k}}\left(X_{\xi_{k}} m_{\alpha}\right)\right)(q, p) d t+\frac{\partial \ell}{\partial q^{i}}(u, q) A_{\alpha}^{i}(q) d t \\
= & \left\{m_{\alpha}(q, p), m_{\beta}(q, p)\right\} d \widehat{x}_{t}^{\beta}+\frac{1}{2}\left\{\left\{m_{\alpha}(q, p), m_{\beta}(q, p)\right\}, m_{\gamma}(q, p)\right\} \sum_{k=1}^{N} \xi_{k}^{\beta} \xi_{k}^{\gamma} d t \\
& +\frac{\partial \ell}{\partial q^{i}}(u, q) A_{\alpha}^{i}(q) d t .
\end{aligned}
$$

In the direct calculation, the Itô contraction is cancelled by a cross term arising from applying the second-order derivative operator $\frac{1}{2} X_{\xi_{k}}\left(X_{\xi_{k}} \cdot\right)$ from equation (3.11) to the quadratic product $m_{\alpha}(q, p)=p_{i} A_{\alpha}^{i}(q)$. This completes the first proof of Corollary 6 .

Second proof of the first equation in (3.7). In the statement of the Corollary [6 the first Itô equation in (3.7) may also be verified by an even more direct calculation than in (3.14), as follows. To simplify notations in the computations below, we again temporarily suppress the dependence of $A_{\alpha}^{i}$ on the semimartingale $q$, of $\frac{\partial \ell}{\partial q^{i}}$ on the semimartingales $(u, q)$, and of $m_{\alpha}(q, p)$ on the semimartingales $(q, p)$. We also suppress the $k$-index.

By equations (2.12), (2.23), the definition (3.3) of $d \widehat{x}_{t}^{\beta}$, the Itô product rule in (3.12), and Theorem 1, we have,

$$
\begin{aligned}
d m_{\alpha} & =\left\{m_{\alpha}, m_{\beta}\right\} d x_{t}^{\beta}+\frac{\partial \ell}{\partial q^{i}} A_{\alpha}^{i} d t \\
d\left(p_{i} A_{\alpha}^{i}\right) & =-p_{j}\left[A_{\alpha}, A_{\beta}\right]^{j} d \widehat{x}_{t}^{\beta}-\frac{1}{2} d\left(p_{j}\left[A_{\alpha}, A_{\beta}\right]^{j} \xi^{\beta}\right) \cdot d W_{t}+\frac{\partial \ell}{\partial q^{i}} A_{\alpha}^{i} d t,
\end{aligned}
$$


where we have substituted the momentum map definition $m_{\alpha}=p_{i} A_{\alpha}^{i}$ from equation (2.11). We have,

$$
\begin{aligned}
d\left(p_{j}\left[A_{\alpha}, A_{\beta}\right]^{j} \xi^{\beta}\right) \cdot d W_{t} & =\left(\left(d p_{j}\right)\left[A_{\alpha}, A_{\beta}\right]^{j}+p_{j} d\left[A_{\alpha}, A_{\beta}\right]^{j}\right) \xi^{\beta} \cdot d W_{t} \\
& =\left(\left(d p_{j}\right)\left[A_{\alpha}, A_{\beta}\right]^{j}+p_{j} \frac{\partial}{\partial q^{l}}\left[A_{\alpha}, A_{\beta}\right]^{j}\left(d q^{l}\right)\right) \xi^{\beta} \cdot d W_{t} \\
& \stackrel{\sqrt[2.24]{=}}{=}\left(-p_{l} \frac{\partial A_{\gamma}^{l}}{\partial q^{j}}\left[A_{\alpha}, A_{\beta}\right]^{j} \xi^{\gamma}+p_{j} \frac{\partial}{\partial q^{l}}\left[A_{\alpha}, A_{\beta}\right]^{j} A_{\gamma}^{l} \xi^{\gamma}\right) \xi^{\beta} d t \\
& =\left(-p_{j}\left[A_{\alpha}, A_{\beta}\right]^{l} \frac{\partial A_{\gamma}^{j}}{\partial q^{l}}+p_{j} A_{\gamma}^{l} \frac{\partial}{\partial q^{l}}\left[A_{\alpha}, A_{\beta}\right]^{j}\right) \xi^{\beta} \xi^{\gamma} d t \\
& =p_{j}\left[A_{\gamma},\left[A_{\alpha}, A_{\beta}\right]\right]^{j} \xi^{\beta} \xi^{\gamma} d t \\
& \stackrel{2.212}{=}\left\{m_{\gamma},\left\{m_{\alpha}, m_{\beta}\right\}\right\} \xi^{\beta} \xi^{\gamma} d t .
\end{aligned}
$$

Consequently, we may write the entire equation (3.15) as (reinstating the $k$-indices)

$$
\begin{aligned}
d m_{\alpha} & =\left\{m_{\alpha}, m_{\beta}\right\} d \widehat{x}_{t}^{\beta}-\frac{1}{2}\left\{m_{\gamma},\left\{m_{\alpha}, m_{\beta}\right\}\right\}\left(\sum_{k=1}^{N} \xi_{k}^{\beta} \xi_{k}^{\gamma}\right) d t+\frac{\partial \ell}{\partial q^{i}} A_{\alpha}^{i} d t \\
& =\left\{m_{\alpha}, m_{\beta}\right\}\left(u^{\beta} d t+\xi_{k}^{\beta} d W_{t}^{k}\right)+\frac{1}{2}\left\{\left\{m_{\alpha}, m_{\beta}\right\}, m_{\gamma}\right\}\left(\sum_{k=1}^{N} \xi_{k}^{\beta} \xi_{k}^{\gamma}\right) d t+\frac{\partial \ell}{\partial q^{i}} A_{\alpha}^{i} d t \\
& =X_{d \widehat{x}_{t}} m_{\alpha}+\frac{1}{2}\left(\sum_{k=1}^{N} x_{\xi_{k}}\left(x_{\xi_{k}} m_{\alpha}\right)\right) d t+\frac{\partial \ell}{\partial q^{i}} A_{\alpha}^{i} d t \quad \text { using the notations (3.1) and (3.9), }
\end{aligned}
$$

in agreement with the first equation in (3.7).

Third proof of the first equation in (3.7). Let $\eta:[0, T] \rightarrow \mathfrak{g}$ be an arbitrary random curve of bounded variation. We begin the third proof by computing

$$
\begin{aligned}
& \left\langle d\left(\frac{\delta \ell}{\delta u}(u, q)\right), \eta\right\rangle=d\left(p_{i} A_{\alpha}^{i}(q)\right) \eta^{\alpha} \\
& =\left(A_{\alpha}^{i}(q) \eta^{\alpha}\right) \circ d p_{i}+\left(p_{i} \frac{\partial A_{\alpha}^{i}}{\partial q^{j}}(q) \eta^{\alpha}\right) \circ d q^{j} \\
& \frac{\sqrt[2.16]{=}}{=} R_{j} \frac{\partial A_{\alpha}^{j}}{\partial q^{i}}(q) u^{\alpha} A_{\beta}^{i}(q) \eta^{\beta} d t-\left(p_{j} \frac{\partial A_{\alpha}^{j}}{\partial q^{i}}(q) \xi_{k}^{\alpha} A_{\beta}^{i}(q) \eta^{\beta}\right) \circ d W_{t}^{k} \\
& +A_{\beta}^{i}(q) \eta^{\beta} \frac{\partial \ell}{\partial q^{i}}(u, q) d t \\
& +p_{i} \frac{\partial A_{\alpha}^{i}}{\partial q^{j}}(q) \eta^{\alpha} A_{\beta}^{j}(q) u^{\beta} d t+\left(p_{i} \frac{\partial A_{\alpha}^{i}}{\partial q^{j}}(q) \eta^{\alpha} A_{\beta}^{j}(q) \xi_{k}^{\beta}\right) \circ d W_{t}^{k} \\
& \stackrel{(2.3)}{=} p_{j}\left(u_{Q}^{i} \frac{\partial \eta_{Q}^{j}}{\partial q^{i}}-\eta_{Q}^{i} \frac{\partial u_{Q}^{j}}{\partial q^{i}}\right)(q) d t+p_{j}\left(\left(\xi_{k}\right)_{Q}^{i} \frac{\partial \eta_{Q}^{j}}{\partial q^{i}}-\eta_{Q}^{i} \frac{\partial\left(\xi_{k}\right)_{Q}^{j}}{\partial q^{i}}\right)(q) \circ d W_{t}^{k} \\
& +A_{\beta}^{i}(q) \eta^{\beta} \frac{\partial \ell}{\partial q^{i}}(u, q) d t \\
& =p_{j}\left[u_{Q}, \eta_{Q}\right]^{j}(q) d t+p_{j}\left[\left(\xi_{k}\right)_{Q}, \eta_{Q}\right]^{j}(q) \circ d W_{t}^{k}+A_{\beta}^{i}(q) \eta^{\beta} \frac{\partial \ell}{\partial q^{i}}(u, q) d t \\
& =p_{j}[u, \eta]_{Q}^{j}(q) d t+p_{j}\left[\xi_{k}, \eta\right]_{Q}^{j}(q) \circ d W_{t}^{k}+A_{\beta}^{i}(q) \eta^{\beta} \frac{\partial \ell}{\partial q^{i}}(u, q) d t .
\end{aligned}
$$

Next, we compute the Itô contraction term, namely the difference between the Stratonovich integral above and the corresponding Itô one. Consequently, we find

$$
d p_{j} \simeq-\left(p_{i} \frac{\partial A_{\alpha}^{i}}{\partial q^{j}}(q) \xi_{k}^{\alpha}\right) d W_{t}^{k}
$$


and

$$
d\left[\xi_{k}, \eta\right]_{Q}^{j}(q)=d\left(A_{\alpha}^{j}(q)\left[\xi_{k}, \eta\right]^{\alpha}(q)\right) \simeq\left(\frac{\partial A_{\alpha}^{j}}{\partial q^{i}}(q)\left[\xi_{k}, \eta\right]^{\alpha}(q) A_{\beta}^{i}(q) \xi_{k}^{\beta}\right) d W_{t}^{k}
$$

Therefore,

$$
\begin{aligned}
d\left(R_{j}\left[\xi_{k}, \eta\right]_{Q}^{j}(q)\right) & \simeq-\left(p_{i} A_{\beta}^{j}(q)\left[\xi_{k}, \eta\right]^{\beta}(q) \frac{\partial A_{\alpha}^{i}}{\partial q^{j}}(q) \xi_{k}^{\alpha}\right) d W_{t}^{k}+\left(p_{j} \frac{\partial A_{\alpha}^{j}}{\partial q^{i}}(q)\left[\xi_{k}, \eta\right]^{\alpha}(q) A_{\beta}^{i}(q) \xi_{k}^{\beta}\right) d W_{t}^{k} \\
& =R_{i}\left(\left(\xi_{k}\right)_{Q}^{j}(q) \frac{\partial\left[\xi_{k}, \eta\right]_{Q}^{i}}{\partial q^{j}}(q)-\left[\xi_{k}, \eta\right]_{Q}^{j}(q) \frac{\partial\left(\xi_{k}\right)_{Q}^{i}}{\partial q^{j}}(q)\right) d W_{t}^{k} \\
& =R_{i}\left[\left(\xi_{k}\right)_{Q},\left[\xi_{k}, \eta\right]_{Q}\right]^{i}(q) d W_{t}^{k} \\
& =R_{i}\left[\xi_{k},\left[\xi_{k}, \eta\right]\right]_{Q}^{i}(q) d W_{t}^{k}
\end{aligned}
$$

and we obtain the Itô contraction term

$$
d\left(\boldsymbol{R}_{j}\left[\xi_{k}, \eta\right]_{Q}^{j}(q)\right) \cdot d W_{t}^{k}=\sum_{k=1}^{N} \boldsymbol{R}_{j}\left[\xi_{k},\left[\xi_{k}, \eta\right]\right]_{Q}^{j}(q) d t=\sum_{k=1}^{N} \boldsymbol{R}_{j}\left(\operatorname{ad}_{\xi_{k}} \operatorname{ad}_{\xi_{k}} \eta\right)_{Q}^{j}(q) d t .
$$

Thus, the first Stratonovich equation in (2.23) reads, in the Itô version,

$$
\begin{aligned}
d\left(\frac{\delta l}{\delta u}(u, q)\right)=- & \operatorname{ad}_{u}^{*}\left(\frac{\delta l}{\delta u}(u, q)\right) d t-\operatorname{ad}_{\xi_{k}}^{*}\left(\frac{\delta l}{\delta u}(u, q)\right) d W_{t}^{k} \\
& +\frac{1}{2} \sum_{k=1}^{N} \operatorname{ad}_{\xi_{k}}^{*} \operatorname{ad}_{\xi_{k}}^{*}\left(\frac{\delta l}{\delta u}(u, q)\right) d t+\frac{\partial \ell}{\partial q^{i}}(u, \boldsymbol{q}) A_{\alpha}^{i}(\boldsymbol{q}) e^{\alpha} \in \mathfrak{g}^{*},
\end{aligned}
$$

which is an explicit version of the first equation in (3.7). This finishes the third proof of Corollary 6 .

\section{Stochastic Hamiltonian formulation}

The goal of this section is to present the Hamiltonian version of Theorem 1 and analyze its consequences.

In Section 2, we found the stochastic equations of motion (2.23) on $\mathfrak{g}^{*} \times T^{*} Q$ and (2.30) on $\mathfrak{g}^{*} \times Q$. We want to deduce these equations in a purely Hamiltonian manner, without any reference to variational principles or the Lagrangian formulation of Sections 2 and 3 . Thus, we need stochastic Hamiltonians $\widetilde{h}\left(m, p_{q}\right)$ and $h(m, q)$. The latter Hamiltonian was already defined in the last paragraph of Section 2 We also need the Poisson brackets on $\mathfrak{g}^{*} \times T^{*} Q$ and $\mathfrak{g}^{*} \times Q$.

\subsection{The deterministic Hamilton equations}

We first recall the Poisson structure on $\mathfrak{g}^{*} \times P$ introduced in [20, where the Lie group $G$, whose Lie algebra is $\mathfrak{g}$, acts on the right on the Poisson manifold $P$ by Poisson diffeomorphisms.

The Poisson manifold $\mathfrak{g}^{*} \times P$. In this paragraph, the entire discussion is non-stochastic. We recall below the results in 20] relevant to our development and expand on it in certain directions we will need later. The framework studied in [20], when adapted to our situation, is the following. Let a Lie group $G$ act on the right by Poisson diffeomorphisms on the Poisson manifold $P$. Endow $T^{*} G \times P$ with the Poisson bracket equal to the sum of the canonical bracket $\{\cdot, \cdot\}$ on $T^{*} G$ and the given Poisson bracket $\{\cdot, \cdot\}_{P}$ on $P$. Define the free proper left $G$-action by Poisson diffeomorphisms on $\left(T^{*} G \times P,\{\cdot, \cdot\}+\{\cdot, \cdot\}_{P}\right)$ by $h \cdot\left(\alpha_{g}, p\right):=\left(T_{h g}^{*} L_{h^{-1}}\left(\alpha_{g}\right), p \cdot h^{-1}\right)$, where $g, h \in G$, $\alpha_{g} \in T_{g}^{*} G, p \in P$, and $p \cdot h^{-1}$ denotes the given right action of $h^{-1}$ on the point $p$. Then the map $\phi: T^{*} G \times P \ni$ 
$\left(\alpha_{g}, p\right) \mapsto\left(T_{e}^{*} L_{g}\left(\alpha_{g}\right), p \cdot g\right) \in \mathfrak{g}^{*} \times P$ is $G$-invariant and induces a diffeomorphism $\phi / G:\left(T^{*} G \times P\right) / G \rightarrow \mathfrak{g}^{*} \times P$. The push forward of the quotient Poisson bracket on $\left(T^{*} G \times P\right) / G$ by $\phi / G$ yields the Poisson bracket

$$
\{f, h\}_{\mathfrak{g}^{*} \times P}(\mu, p)=\left\{f^{p}, h^{p}\right\}_{-}(\mu)+\left\langle\mathbf{d} f^{\mu}(p),\left(\frac{\delta h^{p}}{\delta \mu}\right)_{P}(p)\right\rangle_{P}-\left\langle\mathbf{d} h^{\mu}(p),\left(\frac{\delta f^{p}}{\delta \mu}\right)_{P}(p)\right\rangle_{P}+\left\{f^{\mu}, h^{\mu}\right\}_{P}(p),
$$

for all $f, h \in C^{\infty}\left(\mathfrak{g}^{*} \times P\right)$, where $f^{\mu}, h^{\mu} \in C^{\infty}(P)$ and $f^{p}, h^{p} \in C^{\infty}\left(\mathfrak{g}^{*}\right)$ are defined by $f^{\mu}(p):=f^{p}(\mu):=f(\mu, p)$, for all $\mu \in \mathfrak{g}^{*}, p \in P$, and similarly for $h$ ([20, Proposition 2.1]). Thus, Hamilton's equations for $h \in C^{\infty}\left(\mathfrak{g}^{*} \times P\right)$ are

$$
\frac{d}{d t} \mu=\operatorname{ad}_{\frac{\delta h^{p}}{\delta \mu}}^{*} \mu-\mathbf{J}_{T^{*} P}\left(\mathbf{d} h^{\mu}(p)\right), \quad \frac{d}{d t} p=\left(\frac{\delta h^{p}}{\delta \mu}\right)_{P}(p)+X_{h^{\mu}}^{P}(p)
$$

where $X_{h^{\mu}}^{P}$ denotes the Hamiltonian vector field of $h^{\mu} \in C^{\infty}(P)$ on the Poisson manifold $P$ and $\mathbf{J}_{T^{*} P}: T^{*} P \rightarrow \mathfrak{g}^{*}$ is the momentum map of the cotangent lifted action (see (2.8) with $Q$ replaced by $P$ ).

Suppose now that the right $\mathfrak{g}$-action on $P$ has a momentum map $\mathbf{J}_{P}: P \rightarrow \mathfrak{g}^{*}$, which means that $\xi_{P}[f]=$ $\left\{f, \mathbf{J}_{P}^{\xi}\right\}_{P}$ for all $f \in C^{\infty}(P)$ and all $\xi \in \mathfrak{g}$, where $\mathbf{J}_{P}^{\xi}(p):=\left\langle\mathbf{J}_{P}(p), \xi\right\rangle_{\mathfrak{g}}$. Suppose also that $\mathbf{J}_{P}$ is infinitesimally equivariant i.e., $\mathbf{J}_{P}^{[\xi, \eta]}=-\left\{\mathbf{J}_{P}^{\xi}, \mathbf{J}_{P}^{\eta}\right\}_{P}$, for all $\xi, \eta \in \mathfrak{g}$. We recall that the existence of a momentum map on $P$ for a connected Lie group action forces the group orbits to be included in the symplectic leaves of $P$, which is a rather stringent condition. There are many examples of Poisson Lie group actions that do not admit a momentum map. (See, e.g., [26, Chapters 4 and 5] for a discussion of this problem.) However, in the presence of an equivariant momentum map $\mathbf{J}_{P}: P \rightarrow \mathfrak{g}^{*}$, the diffeomorphism $\psi: \mathfrak{g}^{*} \times P \ni(\mu, p) \mapsto\left(\mu-\mathbf{J}_{P}(p), p\right) \in \mathfrak{g}^{*} \times P$ pushes forward the Poisson bracket $\{\cdot, \cdot\}_{\mathfrak{g}^{*} \times P}$, given by (4.1), to the sum Poisson bracket

$$
\{f, h\}_{\mathrm{sum}}(\mu, p):=\left\{f^{p}, h^{p}\right\}_{-}(\mu)+\left\{f^{\mu}, h^{\mu}\right\}_{P}(p)
$$

on $\mathfrak{g}^{*} \times P$. This is proved for left actions in [20, Proposition 2.2]; although our formulas in (4.1) and the definition of $\psi$ have relative sign changes because we work with a right $G$-action on $P$. The proof is a direct verification. Hamilton's equations $\frac{d}{d t} f=\{f, h\}$ for the sum Poisson bracket (4.3) are

$$
\frac{d}{d t} \mu=\operatorname{ad}_{\frac{\delta h p}{\delta \mu}}^{*} \mu, \quad \frac{d}{d t} p=X_{h^{\mu}}^{P}(p) .
$$

Using (4.3), it follows that if $k \in C^{\infty}\left(\mathfrak{g}^{*} \times P\right)$ is a Casimir function on $\left(\mathfrak{g}^{*} \times P,\{\cdot, \cdot\}_{\text {sum }}\right)$, then $k \circ \psi$ is a Casimir function on $\left(\mathfrak{g}^{*} \times P,\{\cdot, \cdot\}_{\mathfrak{g}^{*} \times P}\right)$. In particular, if $k_{P} \in C^{\infty}(P)$ is a Casimir function, then the function $(\mu, p) \mapsto k_{P}(p)$ is a Casimir function for $\left(\mathfrak{g}^{*} \times P,\{\cdot, \cdot\}_{\mathfrak{g}^{*} \times P}\right)$. This can also be easily checked directly using (4.2). More interestingly, if $k_{\mathfrak{g}^{*}} \in C^{\infty}\left(\mathfrak{g}^{*}\right)$ is a Casimir function on $\mathfrak{g}^{*}$, then $(\mu, p) \mapsto k_{\mathfrak{g}^{*}}\left(\mu-\mathbf{J}_{P}(p)\right)$ is a Casimir function for $\left(\mathfrak{g}^{*} \times P,\{\cdot, \cdot\}_{\mathfrak{g}^{*} \times P}\right)([20$, Corollary 2.3]).

Since the projections $\pi_{\mathfrak{g}^{*}}:\left(\mathfrak{g}^{*} \times P,\{\cdot, \cdot\}_{\text {sum }}\right) \rightarrow \mathfrak{g}_{-}^{*}$ and $\pi_{P}:\left(\mathfrak{g}^{*} \times P,\{\cdot, \cdot\}_{\text {sum }}\right) \rightarrow P$ are Poisson maps, their compositions

$$
\pi_{\mathfrak{g}^{*}} \circ \psi:\left(\mathfrak{g}^{*} \times P,\{\cdot, \cdot\}_{\mathfrak{g}^{*} \times P}\right) \ni(\mu, p) \longmapsto \mu-\mathbf{J}_{P}(p) \in \mathfrak{g}_{-}^{*}, \quad \pi_{P} \circ \psi:\left(\mathfrak{g}^{*} \times P,\{\cdot, \cdot\}_{\mathfrak{g}^{*} \times P}\right) \ni(\mu, p) \longmapsto p \in P
$$

with the Poisson diffeomorphism $\psi:\left(\mathfrak{g}^{*} \times P,\{\cdot, \cdot\}_{\mathfrak{g}^{*} \times P}\right) \rightarrow\left(\mathfrak{g}^{*} \times P,\{\cdot, \cdot\}_{\text {sum }}\right)$ are also Poisson maps.

Remarkably, the projection $\pi_{\mathfrak{g}^{*}}:\left(\mathfrak{g}^{*} \times P,\{\cdot, \cdot\}_{\mathfrak{g}^{*} \times P}\right) \rightarrow \mathfrak{g}_{-}^{*}$ is also a Poisson map, as an easy direct verification shows, using for $\mathrm{f} \in C^{\infty}\left(\mathfrak{g}^{*}\right)$ the identities $\left(\mathrm{f} \circ \pi_{\mathfrak{g}^{*}}\right)^{p}=\mathrm{f}$ for every $p \in P$ and $\left(\mathfrak{f} \circ \pi_{\mathfrak{g}^{*}}\right)^{\mu}=\mathrm{f}(\mu)$, a constant function on $P$, for every $\mu \in \mathfrak{g}^{*}$. In particular, this means that Hamilton's equations (4.2) for a Hamiltonian of the form $h:=\mathrm{h} \circ \pi_{\mathfrak{g}^{*}}$, where $\mathrm{h} \in C^{\infty}\left(\mathfrak{g}^{*}\right)$ (i.e., $h$ does not depend on $p \in P$ ), are the Lie-Poisson equations for $\mathrm{h}$ on $\mathfrak{g}_{-}^{*}$ which completely decouple from the second equation in (4.2). The second equation is given by an infinitesimal generator at every instance of time, namely, if $\mu(t)$ is a solution of the Lie-Poisson equation $\frac{d}{d t} \mu=\operatorname{ad}_{\frac{\delta h}{\delta \mu}}^{*} \mu$, then the second equation in (4.2) is the time-dependent infinitesimal generator equation

$$
\frac{d}{d t} p(t)=\left(\frac{\delta \mathrm{h}}{\delta \mu(t)}\right)_{P}(p(t))
$$


Similarly, the projection $\pi_{P}: \mathfrak{g}^{*} \times P \rightarrow P$ is a Poisson map relative to both Poisson brackets $\{\cdot, \cdot\}_{\mathfrak{g}^{*} \times P}$ and $\{\cdot, \cdot\}_{\text {sum }}$, because if $\overline{\mathrm{f}} \in C^{\infty}(P)$, then $\left(\overline{\mathrm{f}} \circ \pi_{P}\right)^{p}=\overline{\mathrm{f}}(p)$, a constant on $\mathfrak{g}^{*}$, and $\left(\overline{\mathrm{f}} \circ \pi_{P}\right)^{\mu}=\overline{\mathrm{f}}$, for any $\mu \in \mathfrak{g}^{*}$.

Hamilton's equations (4.2) and (4.4) show that the manifolds $\{\mu\} \times P$ and $\mathfrak{g}_{-}^{*} \times\{p\}$ for any $\mu \in \mathfrak{g}^{*}, p \in P$, are not Poisson submanifolds of $\mathfrak{g}^{*} \times P$ endowed with either Poisson bracket $\{\cdot, \cdot\}_{\mathfrak{g}^{*} \times P}$ or $\{\cdot, \cdot\}_{\text {sum }}$.

The Poisson brackets on $\mathfrak{g}^{*} \times Q$ and $\mathfrak{g}^{*} \times T^{*} Q$. We specialize the results of the previous paragraph to the following Poisson manifolds: $Q$, endowed with the zero Poisson structure, and $T^{*} Q$, endowed with the canonical Poisson structure (whose local expression is (2.5)). We continue to work in the non-stochastic context.

For any $f, h \in C^{\infty}\left(\mathfrak{g}^{*} \times Q\right)$, the Poisson bracket (4.1) reads

$$
\begin{aligned}
\{f, h\}_{\mathfrak{g}_{-}^{*} \times Q}(m, q): & =\left[\begin{array}{c}
\partial f / \partial m_{\alpha} \\
\partial f / \partial q^{i}
\end{array}\right]^{\top}\left[\begin{array}{cc}
-c_{\alpha \beta^{\gamma}} m_{\gamma} & -A_{\alpha}^{j} \\
A_{\beta}^{i} & 0
\end{array}\right]\left[\begin{array}{c}
\partial h / \partial m_{\beta} \\
\partial h / \partial q^{j}
\end{array}\right] \\
& =\left\{f^{q}, h^{q}\right\}_{-}(m)+\left\langle\mathbf{d} f^{m}(q),\left(\frac{\delta h^{q}}{\delta m}\right)_{Q}(q)\right\rangle_{Q}-\left\langle\mathbf{d} h^{m}(q),\left(\frac{\delta f^{q}}{\delta m}\right)_{Q}(q)\right\rangle_{Q},
\end{aligned}
$$

where $f^{q} \in C^{\infty}\left(\mathfrak{g}^{*}\right)$ and $f^{\mu} \in C^{\infty}(Q)$ are defined by $f^{q}(\mu):=f^{m}(q):=f(m, q)$, for all $m \in \mathfrak{g}^{*}, q \in Q$, and $\{\cdot, \cdot\}_{-}$ is the minus Lie-Poisson bracket (2.13) on $\mathfrak{g}_{-}^{*}$.

Similarly, for any $\widetilde{f}, \widetilde{h} \in C^{\infty}\left(\mathfrak{g}^{*} \times T^{*} Q\right)$, the Poisson bracket (4.1) reads

$$
\begin{aligned}
& \{\widetilde{f}, \widetilde{h}\}_{\mathfrak{g}_{-}^{*} \times T^{*} Q}\left(m, p_{q}\right):=\left[\begin{array}{c}
\partial \widetilde{f} / \partial m_{\alpha} \\
\partial \widetilde{f} / \partial q^{i} \\
\partial \widetilde{f} / \partial p_{i}
\end{array}\right]^{\top}\left[\begin{array}{ccc}
-m_{[\alpha, \beta]} & -A_{\alpha}^{j} & p_{k} \frac{\partial A_{\alpha}^{k}}{\partial q^{j}} \\
A_{\beta}^{i} & 0 & \delta_{j}^{i} \\
-p_{k} \frac{\partial A_{\beta}^{k}}{\partial q^{i}} & -\delta_{i}^{j} & 0
\end{array}\right]\left[\begin{array}{c}
\partial \widetilde{h} / \partial m_{\beta} \\
\partial \widetilde{h} / \partial q^{j} \\
\partial \widetilde{h} / \partial p_{j}
\end{array}\right] \\
& =\left\{\widetilde{f}^{p_{q}}, \widetilde{h}^{p_{q}}\right\}_{-}(m)+\left\langle\mathbf{d} f^{m}\left(p_{q}\right),\left(\frac{\delta \widetilde{h}^{p_{q}}}{\delta m}\right)_{T^{*} Q}\left(p_{q}\right)\right\rangle_{Q}-\left\langle\mathbf{d} h^{m}\left(p_{q}\right),\left(\frac{\delta \widetilde{f}^{p_{q}}}{\delta m}\right)_{T^{*} Q}\left(p_{q}\right)\right\rangle_{Q}+\left\{\widetilde{f}^{m}, \widetilde{h}^{m}\right\}\left(p_{q}\right),
\end{aligned}
$$

where $\widetilde{f}^{p_{q}} \in C^{\infty}\left(\mathfrak{g}^{*}\right)$ and $\widetilde{f}^{m} \in C^{\infty}\left(T^{*} Q\right)$ are defined by $\widetilde{f}^{p_{q}}(m):=\widetilde{f}^{m}\left(p_{q}\right):=\widetilde{f}\left(m, p_{q}\right)$, for all $m \in \mathfrak{g}^{*}, p_{q} \in T^{*} Q$, and $\{\cdot, \cdot\}$ is the canonical Poisson bracket (2.5) on $T^{*} Q$. This proves the first statement in the following theorem.

Theorem 8. The brackets (4.7) and (4.8) are Poisson brackets on $\mathfrak{g}^{*} \times Q$ and $\mathfrak{g}^{*} \times T^{*} Q$, respectively. Hamilton's equations on $\mathfrak{g}^{*} \times T^{*} Q$ are given by (4.2) with $P$ replaced by $T^{*} Q$. In standard coordinates, for $\widetilde{h} \in C^{\infty}\left(\mathfrak{g}^{*} \times T^{*} Q\right)$, these equations are

$$
\frac{d}{d t} m_{\alpha}=-m_{[\alpha, \beta]} \frac{\partial \widetilde{h}}{\partial m_{\beta}}-A_{\alpha}^{j} \frac{\partial \widetilde{h}}{\partial q^{j}}+p_{k} \frac{\partial A_{\alpha}^{k}}{\partial q^{j}} \frac{\partial \widetilde{h}}{\partial p_{j}}, \quad \frac{d}{d t} q^{i}=A_{\beta}^{i} \frac{\partial \widetilde{h}}{\partial m_{\beta}}+\frac{\partial \widetilde{h}}{\partial p_{i}}, \quad \frac{d}{d t} p_{i}=-p_{k} \frac{\partial A_{\beta}^{k}}{\partial q^{i}} \frac{\partial \widetilde{h}}{\partial m_{\beta}}-\frac{\partial \widetilde{h}}{\partial q^{i}} .
$$

The diffeomorphism $\psi: \mathfrak{g}^{*} \times T^{*} Q \ni\left(\mu, p_{q}\right) \mapsto\left(\mu-\mathbf{J}_{T^{*} Q}(p), p_{q}\right) \in \mathfrak{g}^{*} \times T^{*} Q$ pushes forward the Poisson bracket (4.8) to the sum Poisson bracket. If $k_{\mathfrak{g}^{*}} \in C^{\infty}\left(\mathfrak{g}^{*}\right)$ is a Casimir function on $\mathfrak{g}^{*}$, then $\left(\mu, p_{q}\right) \mapsto k_{\mathfrak{g}^{*}}\left(\mu-\mathbf{J}_{T^{*} Q}\left(p_{q}\right)\right)$ is a Casimir function for $\left(\mathfrak{g}^{*} \times T^{*} Q,\{\cdot, \cdot\}_{\mathfrak{g}^{*} \times T^{*} Q}\right)$.

Hamilton's equations (4.2) on $\mathfrak{g}^{*} \times Q$ for $h \in C^{\infty}\left(\mathfrak{g}^{*} \times Q\right)$ (with $P$ replaced by the trivial Poisson manifold $Q$ ) are Hamel's equations [15]: 3

$$
\begin{array}{ll}
\frac{d}{d t} m=\operatorname{ad}_{\frac{\delta h q}{\delta m}}^{*} m-\mathbf{J}_{T^{*} Q}\left(\mathbf{d} h^{m}(q)\right), & \frac{d}{d t} q=\left(\frac{\delta h^{q}}{\delta m}\right)_{Q}(q) \\
\frac{d}{d t} m_{\alpha}=-c_{\alpha \beta} m_{\gamma} \frac{\partial h}{\partial m_{\beta}}-A_{\alpha}^{j} \frac{\partial h}{\partial q^{j}}, & \frac{d}{d t} q^{i}=A_{\beta}^{i} \frac{\partial h}{\partial m_{\beta}} .
\end{array}
$$

If $\widetilde{h}$ does not depend on $p_{q} \in T^{*} Q$ (respectively, $h$ does not depend on $q \in Q$ ), then Hamilton's equations (4.9) (respectively, (4.10) ) decouple into the Lie-Poisson equations on $\mathfrak{g}_{-}^{*}$ and the time-dependent infinitesimal generator equations for $\frac{\delta \widetilde{h}}{\delta m(t)} \in \mathfrak{g}$ on $T^{*} Q$ (respectively, $\frac{\delta h}{\delta m(t)} \in \mathfrak{g}$ on $Q$ ).

\footnotetext{
${ }^{3}$ For a modern formulation, see, e.g., [6, $\S 3.8$, p.144] or [7]
} 
The four projections of $\mathfrak{g}^{*} \times Q$ and $\mathfrak{g}^{*} \times T^{*} Q$ on every factor are Poisson ( $\mathfrak{g}^{*}$ has the minus Lie-Poisson structure). The map $\mathfrak{g}^{*} \times T^{*} Q \ni\left(\mu, p_{q}\right) \mapsto \mu-\mathbf{J}_{T^{*} Q}\left(p_{q}\right) \in \mathfrak{g}_{-}^{*}$ is Poisson. The embedding $\mathfrak{g}^{*} \times Q \ni(m, q) \mapsto\left(m, 0_{q}\right) \in \mathfrak{g}^{*} \times T^{*} Q$ is not Poisson. The map $\rho: \mathfrak{g}^{*} \times T^{*} Q \ni\left(m, p_{q}\right) \longmapsto(m, q) \in \mathfrak{g}^{*} \times Q$ is Poisson.

Proof. Formulas (4.9) and (4.10) are obtained by calculating (4.2) for these two cases. The statements about the Poisson character of the five projections and the diffeomorphism $\psi$, the decoupling of the equations for Hamiltonians depending only on $m \in \mathfrak{g}^{*}$, as well as the assertion about the Casimir functions, were proved in the previous paragraph for a general Poisson manifold $P$.

Setting all coordinates $p_{i}=0$ in (4.9) does not yield (4.10), i.e., a Hamiltonian vector field on the Poisson manifold $\mathfrak{g}^{*} \times T^{*} Q$, restricted to $\mathfrak{g}^{*} \times Q$ is, in general, not tangent to $\mathfrak{g}^{*} \times Q$. This proves that $\mathfrak{g}^{*} \times Q$ is not a Poisson submanifold of $\mathfrak{g}^{*} \times T^{*} Q$.

Let $\pi: T^{*} Q \rightarrow Q$ be the cotangent bundle projection. The map, $\rho: \mathfrak{g}^{*} \times T^{*} Q \rightarrow \mathfrak{g}^{*} \times Q$ is Poisson. This is a direct verification using the formulas $(f \circ \rho)^{m}=f^{m} \circ \pi, \mathbf{d}(f \circ \rho)^{m}\left(p_{q}\right)=\mathbf{d} f^{m}(q) \circ T_{p_{q}} \pi,(f \circ \rho)^{p_{q}}=f^{q}$, $\frac{\delta(f \circ \rho)^{p_{q}}}{\delta m}=\frac{\delta f^{q}}{\delta m}$, and the fact that the infinitesimal generators $u_{T^{*} Q}$ (of the lifted $G$-action on $T^{*} Q$ ) and $u_{Q}$ (of the $G$-action on $Q$ ) are $\pi$-related.

The last statement is obtained by setting $\partial h / \partial p_{i}=0$ in (4.9).

Remark 9. [Collective Lie-Poisson momentum map dynamics] In (4.10), note that if the Hamiltonian depends only on $m \in \mathfrak{g}^{*}$, i.e., the Hamiltonian is of the form $h:=\mathrm{h} \circ \pi_{\mathfrak{g}^{*}}$, where $\mathrm{h} \in C^{\infty}\left(\mathfrak{g}^{*}\right)$ and $\pi_{\mathfrak{g}^{*}}:\left(\mathfrak{g}^{*} \times Q,\{\cdot, \cdot\}_{\mathfrak{g}^{*} \times Q}\right) \ni$ $(m, q) \mapsto m \in\left(\mathfrak{g}^{*},\{\cdot, \cdot\}_{-}\right)$, Hamel's equations in (4.10) become the Lie-Poisson equations on $\mathfrak{g}_{-}^{*}$. In this case, we say the motion collectivizes (see [14]) since $\pi_{\mathfrak{g}^{*}}$ is a Poisson map.

Remark 10. The manifold $Q$ endowed with the zero Poisson structure does not admit a momentum map $\mathbf{J}_{Q}$ : $Q \rightarrow \mathfrak{g}^{*}$. Indeed, if $\mathbf{J}_{Q}$ existed, we would have $\xi_{Q}[f]=\left\{f, \mathbf{J}_{Q}^{\xi}\right\}_{Q}=0$ for all $f \in C^{\infty}(Q)$ and all $\xi \in \mathfrak{g}$, which would imply the false statement that all smooth functions on $Q$ are $\mathfrak{g}$-invariant. As a consequence, the statement in the previous paragraph about the Poisson bracket on $\mathfrak{g}^{*} \times Q$ being isomorphic to the sum Poisson bracket, which in this case would be just the minus Lie-Poisson bracket, does not apply. Similarly, Casimir functions on $\mathfrak{g}^{*}$ do not induce Casimir functions on $\mathfrak{g}^{*} \times Q$.

For the statement of the next corollary, we need to introduce the fiber translation vector field $T_{\alpha} \in \mathfrak{X}\left(T^{*} Q\right)$ associated to a one-form $\alpha \in \Omega^{1}(Q)$. The map $T^{*} Q \ni p_{q} \mapsto p_{q}-t \alpha(q) \in T^{*} Q, t \in \mathbb{R}$, is a one-parameter group. Define $T_{\alpha}$ to be the vector field with this flow, i.e.,

$$
T_{\alpha}\left(p_{q}\right):=\left.\frac{d}{d t}\right|_{t=0}\left(p_{q}-t \alpha(q)\right) \in T_{p_{q}}\left(T^{*} Q\right) .
$$

This vector field is identical to the vertical lift operation by $-\alpha \in \Omega^{1}\left(T^{*} Q\right)$.

Corollary 11. Hamilton's equations (4.2) (with $\left.P=T^{*} Q\right)$ on $\mathfrak{g}^{*} \times T^{*} Q$ for $\widetilde{h}:=h \circ \rho$, where $h \in C^{\infty}\left(\mathfrak{g}^{*} \times Q\right)$, i.e., $\widetilde{h}\left(m, p_{q}\right):=h(m, q)$, take the form

$$
\frac{d}{d t} m=\operatorname{ad}_{\frac{\delta h q}{\delta m}}^{*} m-\mathbf{J}_{T^{*} Q}\left(\mathbf{d} h^{m}(q)\right), \quad \frac{d}{d t} p_{q}=\left(\frac{\delta h^{q}}{\delta m}\right)_{T^{*} Q}+T_{\mathbf{d} h^{m}}\left(p_{q}\right) .
$$

In addition, equations (4.11) imply both (4.10) and the non-homogeneous Lie-Poisson equations

$$
\frac{d}{d t} \mathbf{J}_{T^{*} Q}\left(p_{q}(t)\right)=\operatorname{ad}_{\frac{\delta h q(t)}{\delta m(t)}}^{*} \mathbf{J}_{T^{*} Q}\left(p_{q}(t)\right)-\mathbf{J}_{T^{*} Q}\left(\mathbf{d} h^{m(t)}(q(t))\right.
$$

for $\mathbf{J}_{T^{*} Q}\left(p_{q}(t)\right)$, where $(m(t), q(t))$ is the solution of Hamel's equations 4.10).

Proof. We have $\widetilde{h}^{p_{q}}=h^{q} \in C^{\infty}\left(\mathfrak{g}^{*}\right)$ and $\widetilde{h}^{m}=h^{m} \circ \pi \in C^{\infty}\left(T^{*} Q\right)$, where $\pi: T^{*} Q \rightarrow Q$ is the cotangent bundle projection. 
We compute $\mathbf{J}_{T^{*}\left(T^{*} Q\right)}\left(\mathbf{d}\left(h^{m} \circ \pi\right)\left(p_{q}\right)\right)$, the second summand on the right hand side of the first equation in (4.2) for $P=T^{*} Q$. To do this, we note that since the $G$-action on $T^{*} Q$ is the cotangent lifted $G$-action on $Q$, the cotangent bundle projection $\pi: T^{*} Q \rightarrow Q$ is equivariant and thus the infinitesimal generators of the two actions for the same Lie algebra element are $\pi$-related, i.e., $T \pi \circ v_{T^{*} Q}=v_{Q} \circ \pi$ for any $v \in \mathfrak{g}$. Therefore,

$$
\begin{aligned}
\left\langle\mathbf{J}_{T^{*}\left(T^{*} Q\right)}\left(\mathbf{d}\left(h^{m} \circ \pi\right)\left(p_{q}\right)\right), v\right\rangle_{\mathfrak{g}} \stackrel{\stackrel{2.8}{=}}{=}\left\langle\mathbf{d}\left(h^{m} \circ \pi\right)\left(p_{q}\right), v_{T^{*} Q}\left(p_{q}\right)\right\rangle_{\mathfrak{g}}=\left\langle\mathbf{d} h^{m}(q), T_{p_{q}} \pi\left(v_{T^{*} Q}\left(p_{q}\right)\right)\right\rangle_{\mathfrak{g}} \\
=\left\langle\mathbf{d} h^{m}(q), v_{Q}(q)\right\rangle_{\mathfrak{g}} \stackrel{2.8}{=}\left\langle\mathbf{J}_{T^{*} Q}\left(\mathbf{d} h^{m}(q)\right), v\right\rangle_{\mathfrak{g}} .
\end{aligned}
$$

Next, we compute $X_{\widetilde{h}^{m}}^{T^{*} Q}\left(p_{q}\right)=X_{h^{m} \circ \pi}^{T^{*} Q}\left(p_{q}\right)$, the second summand on the right hand side of the second equation in (4.2) for $P=T^{*} Q$. Since this affects only the dynamics on $T^{*} Q$, we prove, in general, that

$$
X_{k \circ \pi}^{T^{*} Q}=T_{\mathbf{d} k}, \quad \text { for all } \quad k \in C^{\infty}(Q) .
$$

To prove (4.14), it is easier to work in local coordinates. Hamilton's equations for $k \circ \pi$ are

$$
\frac{d q^{i}}{d t}=\frac{\partial(k \circ \pi)}{\partial p_{i}}=0, \quad \frac{d p_{i}}{d t}=-\frac{\partial(k \circ \pi)}{\partial q^{i}}=-\frac{\partial k}{\partial q^{i}},
$$

whose solution is $q^{i}(t)=q_{0}^{i}, p_{i}(t)=p_{i}^{0}-t \frac{\partial k}{\partial q^{i}}\left(q_{0}\right)$, where $\left(q_{0}^{1}, \ldots, q_{0}^{n}, p_{1}^{0}, \ldots p_{n}^{0}\right)$ is the initial condition. Thus, the flow of $X_{k \circ \pi}^{T^{*} Q}$ is $p_{q} \mapsto p_{q}-t \mathbf{d} k(q)$ which coincides with the flow of $T_{\mathbf{d} k}$, thereby proving (4.14).

Using the identities (4.13) and (4.14), equations (4.2) become (4.11).

Since $\rho:\left(\mathfrak{g}^{*} \times T^{*} Q,\{\cdot, \cdot\}_{\mathfrak{g}^{*} \times T^{*} Q}\right) \ni\left(m, p_{q}\right) \longmapsto(m, q) \in\left(\mathfrak{g}^{*} \times Q,\{\cdot, \cdot\}_{\mathfrak{g}^{*} \times Q}\right)$ is a Poisson map by Theorem 8 , we have $T \rho \circ X_{h \circ \rho}^{\mathfrak{g}^{*} \times T^{*} Q}=X_{h}^{\mathfrak{g}^{*} \times Q} \circ \rho$ for any $h \in C^{\infty}\left(\mathfrak{g}^{*} \times Q\right)$, which is equivalent to saying that (4.11) (the equations of motion defined by the Hamiltonian vector field $X_{h \circ \rho}^{\mathfrak{g}^{*} \times T^{*} Q}$ ) project to (4.10) (the equations of motion defined by the Hamiltonian vector field $\left.X_{h}^{\mathfrak{g}^{*} \times Q}\right)$.

Finally, we prove (4.12). We have

$$
\begin{aligned}
\frac{d}{d t} \mathbf{J}_{T^{*} Q}\left(p_{q}(t)\right) & =T_{p_{q}(t)} \mathbf{J}_{T^{*} Q}\left(\frac{d}{d t} p_{q}(t)\right) \stackrel{4.11}{=} T_{p_{q}(t)} \mathbf{J}_{T^{*} Q}\left(\left(\frac{\delta h^{q(t)}}{\delta m(t)}\right)_{T^{*} Q}\left(p_{q}(t)\right)\right)+T_{p_{q}(t)} \mathbf{J}_{T^{*} Q}\left(T_{\mathbf{d} h^{m}(t)}\left(p_{q}(t)\right)\right) \\
& \frac{2.10}{=} \operatorname{ad}_{\frac{\delta h q(t)}{\delta m(t)}}^{*} \mathbf{J}_{T^{*} Q}\left(p_{q}(t)\right)+T_{p_{q}(t)} \mathbf{J}_{T^{*} Q}\left(X_{h^{m(t)} \circ \pi}^{T^{*} Q}\left(p_{q}(t)\right)\right) .
\end{aligned}
$$

One may compute the second summand by pairing it with any $v \in \mathfrak{g}$ to obtain

$$
\begin{aligned}
\left\langle T_{p_{q}(t)} \mathbf{J}_{T^{*} Q}\left(X_{h^{m}(t) \circ \pi}^{T^{*} Q}\left(p_{q}(t)\right)\right), v\right\rangle_{\mathfrak{g}} & =\left\langle\mathbf{d} \mathbf{J}_{T^{*} Q}^{v}\left(p_{q}(t)\right), X_{h^{m(t)} \circ \pi}^{T^{*} Q}\left(p_{q}(t)\right)\right\rangle_{T^{*} Q}=\left\{\mathbf{J}_{T^{*} Q}^{v}, h^{m(t)} \circ \pi\right\}\left(p_{q}(t)\right) \\
& =-\left\{h^{m(t)} \circ \pi, \mathbf{J}_{T^{*} Q}^{v}\right\}\left(p_{q}(t)\right)=-\left\langle\mathbf{d}\left(h^{m(t)} \circ \pi\right)\left(p_{q}(t)\right), X_{\mathbf{J}_{T^{*} Q}^{v} Q}^{T^{*}}\left(p_{q}(t)\right)\right\rangle_{Q} \\
& \stackrel{2.7}{=}-\left\langle\mathbf{d}\left(h^{m(t)} \circ \pi\right)\left(p_{q}(t)\right), v_{T^{*} Q}\left(p_{q}(t)\right)\right\rangle_{Q} \\
& \stackrel{2.10}{=}-\left\langle\mathbf{d} h^{m(t)}(q(t)), v_{Q}(q(t))\right\rangle_{Q} \\
& \stackrel{2.8}{=}-\left\langle\mathbf{J}_{T^{*} Q}\left(\mathbf{d} h^{m(t)}(q(t)), v\right\rangle_{\mathfrak{g}}\right.
\end{aligned}
$$

Formulas (4.15) and (4.16) now yield (4.12).

Corollary 12. If the Hamiltonian $\widetilde{h} \in C^{\infty}\left(\mathfrak{g}^{*} \times T^{*} Q\right)$ is of the form $\widetilde{h}=h \circ \rho$ and $h \in C^{\infty}\left(\mathfrak{g}^{*} \times Q\right)$ is hyperregular, i.e., the parameter dependent reduced Legendre transformation $\mathfrak{g} \times Q \ni(u, q) \mapsto(m(u, q), q):=\left(\frac{\delta \bar{\ell}^{q}}{\delta u}, q\right) \in \mathfrak{g}^{*} \times Q$ is a diffeomorphism, where $\bar{\ell} \in C^{\infty}(\mathfrak{g} \times Q)$ and $h(m, q):=\langle m, u(m, q)\rangle_{\mathfrak{g}}-\bar{\ell}(u(m, q)$, $q)$, equation (4.12) takes the form

$$
\frac{d}{d t} \mathbf{J}_{T^{*} Q}\left(p_{q}(t)\right)=\operatorname{ad}_{u(t)}^{*} \mathbf{J}_{T^{*} Q}\left(p_{q}(t)\right)+\mathbf{J}_{T^{*} Q}\left(\mathbf{d} \ell^{u(t)}(q(t))\right.
$$


where $(u(t), q(t))$ is the solution of the Lagrangian version of Hamel's equations

$$
\begin{array}{lll}
\frac{d}{d t} \frac{\delta \bar{\ell}^{q}}{\delta u}=\operatorname{ad}_{u}^{*} \frac{\delta \bar{\ell}^{q}}{\delta u}+\mathbf{J}_{T^{*} Q}\left(\mathbf{d} \bar{\ell}^{u}(q)\right), & \frac{d}{d t} q=u_{Q}(q) & \Longleftrightarrow \\
\frac{d}{d t} \frac{\partial \bar{\ell}^{q}}{\partial u^{\alpha}}=-c_{\alpha \beta} \gamma \frac{\partial \bar{\ell}^{q}}{\partial u^{\gamma}} u^{\beta}+A_{\alpha}^{j} \frac{\partial \bar{\ell}}{\partial q^{j}}, & \frac{d}{d t} q^{i}=A_{\beta}^{i} u^{\beta} . &
\end{array}
$$

Proof. By hyperregularity, we can solve for $u$ to get $u(m, q) \in \mathfrak{g}$ and we have

$$
\left\langle\delta m, \frac{\delta h^{q}}{\delta m}\right\rangle_{\mathfrak{g}}=\langle\delta m, u(m, q)\rangle_{\mathfrak{g}}+\left\langle m, \mathbf{D} u^{q}(m) \cdot \delta m\right\rangle_{\mathfrak{g}}-\left\langle\frac{\delta \bar{\ell}^{q}}{\delta u(m, q)}, \mathbf{D} u^{q}(m) \cdot \delta m\right\rangle_{\mathfrak{g}}=\langle\delta m, u(m, q)\rangle_{\mathfrak{g}}
$$

because $\frac{\delta \bar{\ell}^{q}}{\delta u(m, q)}=m$ by definition of $m$ and hyperregularity. Thus $u(m, q)=\frac{\delta h^{q}}{\delta m}$. Since $\bar{\ell}(u, q)=\langle m(u, q), u\rangle_{\mathfrak{g}}-$ $h(m(u, q), q)$, we get

$$
\mathbf{d} \ell^{u}(q)=\left\langle\mathbf{d} m^{u}(q), u\right\rangle_{\mathfrak{g}}-\left\langle\mathbf{d} m^{u}(q), \frac{\delta h^{q}}{\delta m(u, q)}\right\rangle_{\mathfrak{g}}-\mathbf{d} h^{m(u, q)}(q)=-\mathbf{d} h^{m(u, q)}(q),
$$

since, as we just saw and invoking hyperregularity, we have $\frac{\delta h^{q}}{\delta m(u, q)}=u$. Equations (4.17) and (4.18) now follow from (4.12) and (4.10), respectively.

\subsection{The stochastic Hamilton equations}

The Stratonovich stochastic Hamilton equations for semimartingales. We begin by defining Stratonovich stochastic Hamilton equations. Let $(P,\{\cdot, \cdot\})$ be a Poisson manifold. For any $f \in C^{\infty}(P)$, form the semimartingale $f(p(t))$ obtained by replacing the point $p \in P$ by a $P$-valued semimartingale $p(t)$. Consider a semimartingale $\mathscr{Y}_{t}(p):=y_{0}+\int_{0}^{t} \phi_{\alpha}(p(s)) \xi_{k}^{\alpha} \circ d W_{s}^{k}+\int_{0}^{t} \psi(p(s)) d s$, where $\phi_{\alpha}, \psi \in C^{\infty}(P)$ are deterministic smooth functions and $\xi_{k}:=\xi_{k}^{\alpha} e_{\alpha} \in \mathfrak{g}$ are constant elements.

In analogy with Section 2, the (Stratonovich) stochastic Poisson bracket is defined by

$$
\left\{f(p(t)), \circ d_{t} \mathscr{Y}_{t}\right\}:=\left\{f(p(t)), \phi_{\alpha}(p(t))\right\} \xi_{k}^{\alpha} \circ d W_{t}^{k}+\{f(p(t)), \psi(p(t))\} d t .
$$

where

$$
\{f(p(t)), \phi(p(t))\}=\{f, \phi\}(p(t)) .
$$

Definition 13. The (Stratonovich) stochastic Hamilton equations for P-valued semimartingales with stochastic semimartingale Hamiltonian $\mathscr{Y}_{t}(p):=\mathscr{Y}_{0}+\int_{0}^{t} \phi_{\alpha}(p(s)) \xi_{k}^{\alpha} \circ d W_{s}^{k}+\int_{0}^{t} \psi(p(s)) d s$ are

$$
d_{t} f(p(t))=\left\{f(p(t)), \circ d_{t} \mathscr{Y}_{t}\right\}:=\left\{f, \phi_{\alpha}\right\}(p(t)) \xi_{k}^{\alpha} \circ d W_{t}^{k}+\{f, \psi\}(p(t)) d t, \quad \text { for any } \quad f \in C^{\infty}(P),
$$

where the Poisson bracket semimartingales on the right hand side are defined in (2.20) for variations as in (2.19).

If $\left(p^{1}, \ldots, p^{n}\right)$ are coordinates on $P$, the Stratonovich stochastic Hamilton equations thus take the form

$$
d_{t} R^{i}(t)=\left\{p^{i}(t), \circ d_{t} \mathscr{Y}_{t}\right\}=\left\{p^{i}, \phi_{\alpha}\right\}(p(t)) \xi_{k}^{\alpha} \circ d W_{t}^{k}+\left\{p^{i}, \psi\right\}(p(t)) d t .
$$

Let $k \in C^{\infty}(P)$ be a Casimir function. Then, for the semimartingale $k(p(t))$ we have, by Itô's formula,

$$
d_{t} k(p(t))=\left\{k(p(t)), \circ d_{t} \mathcal{Y}\right\}=\left\{k, \phi_{\alpha}\right\}(p(t)) \xi_{k}^{\alpha} \circ d W_{t}^{k}+\{k, \psi\}(p(t)) d t=0,
$$

i.e., the semimartingale $k(p(t))$ is conserved along the stochastic flow of the stochastic Hamiltonian semimartingale $\mathscr{Y}_{t}(p)$. Clearly, $k(p(t))$ is also conserved in the Itô representation. 
The Stratonovich stochastic Hamilton equations on $\mathfrak{g}^{*} \times Q$ and $\mathfrak{g}^{*} \times T^{*} Q$. We continue to denote the semimartingales $q^{i}(t):=q^{i}(q(t), p(t))$ and $p_{i}(t):=p_{i}(q(t), p(t))$. With this definition, the information in Theorem 8, in particular, having the Poisson bracket (4.7) on $\mathfrak{g}^{*} \times Q$, we form the semimartingale

$$
d_{t} h_{t}=\left(h^{1}\right)_{\alpha}(m(q(t), p(t)), q(t)) \xi_{k}^{\alpha} \circ d W_{t}^{k}+h^{2}(m(q(t), p(t)), q(t)) d t,
$$

where $\left(h^{1}\right)_{\alpha}, h^{2} \in C^{\infty}\left(\mathfrak{g}^{*} \times Q\right)$ and $\xi_{k}=\xi_{k}^{\alpha} e_{\alpha} \in \mathfrak{g}$. By (4.20), the Stratonovich stochastic Hamilton equations are

$$
\begin{aligned}
d_{t} m_{\alpha}(q(t), p(t)) & =\left\{m_{\alpha}(q(t), p(t)), \circ d_{t} h_{t}(m(q(t), p(t)), q(t))\right\}_{\mathfrak{g}^{*} \times Q} \\
d_{t} q^{i}(t) & =\left\{q^{i}(t), \circ d_{t} h_{t}(m(q(t), p(t)), q(t))\right\}_{\mathfrak{g}^{*} \times Q} .
\end{aligned}
$$

That is,

$$
\begin{aligned}
d_{t} m_{\alpha}(q(t), p(t))=\{ & \left.m_{\alpha}(q(t), p(t)), \circ d_{t} h_{t}(m(q(t), p(t)), q(t))\right\}_{\mathfrak{g}^{*} \times Q} \\
=\{ & \left.m_{\alpha}(q(t), p(t)), m_{\beta}(q(t), p(t))\right\}_{\mathfrak{g}^{*} \times Q} \circ d_{t}\left(\frac{\partial h_{t}}{\partial m_{\beta}}\right)((q(t), p(t)), q(t)) \\
& \quad+\left\{m_{\alpha}(q(t), p(t)), q^{j}(t)\right\}_{\mathfrak{g}^{*} \times Q} \circ d_{t}\left(\frac{\partial h_{t}}{\partial q^{j}}\right)((q(t), p(t)), q(t)) \\
=- & c_{\alpha \beta^{\gamma}} m_{\gamma}(q(t), p(t)) \circ d_{t}\left(\frac{\partial h_{t}}{\partial m_{\beta}}\right)((q(t), p(t)), q(t)) \\
& \quad-A_{\alpha}^{j}(q(t)) \circ d_{t}\left(\frac{\partial h_{t}}{\partial q^{j}}\right)((q(t), p(t)), q(t)) \\
=: & \left.\operatorname{ad}_{d\left(\frac{\delta \hbar_{t}}{\partial m}\right)((q(t), p(t)), q(t))} m(q(t), p(t))\right]-A_{\alpha}^{j}(q(t)) \circ d_{t}\left(\frac{\partial h_{t}}{\partial q^{j}}\right)((q(t), p(t)), q(t)), \\
d_{t} q^{i}(t)=\left\{q^{i}(t), \circ d_{t} h_{t}(m(q(t), p(t)), q(t))\right\}_{\mathfrak{g}^{*} \times Q} & \left\{q^{i}(t), m_{\beta}(q(t), p(t))\right\}_{\mathfrak{g}^{*} \times Q} \circ d\left(\frac{\partial h_{t}}{\partial m_{\beta}}\right)((q(t), p(t)), q(t)) \\
= & A_{\beta}^{i}(q(t)) \circ d\left(\frac{\partial h_{t}}{\partial m_{\beta}}\right)((q(t), p(t)), q(t)),
\end{aligned}
$$

which are identical to the stochastic equations of motion (2.30) in Theorem 4 once we observe that for the functional $h_{t}$ considered there, the explicit $q$-dependence comes only from its bounded variation part (defined by $h^{2}$ ) and therefore $\circ d_{t}\left(\frac{\partial h_{t}}{\partial q^{j}}\right)=\left(\frac{\partial h_{t}}{\partial q^{j}}\right) d t$.

Note that equations (4.22) comprise the stochastic version of Hamel's equations (4.10). As in the deterministic case (see Remark 9), note that if $h_{t}$ depends only on the $\mathfrak{g}^{*}$-valued semimartingale $m(q(t), p(t)$ ), then equations (4.22) decouple into the stochastic Lie-Poisson equations on $\mathfrak{g}_{-}^{*}$ and the stochastic infinitesimal generator equation for $\frac{\delta h_{t}}{\delta m}((q(t), p(t)), q(t)) \in \mathfrak{g}$.

Our goal is to derive (4.22) purely from a Hamiltonian point of view and, similarly, Stratonovich stochastic Hamilton equations on $\mathfrak{g}^{*}$ to $T^{*} Q$. In particular, this means that the semimartingale $m(q(t), p(t))$ needs to be replaced by a semimartingale $m$ in order not to appeal to the Legendre transformation of the Lagrangian $\ell$. So, the setup is the following general situation.

Let $h_{t}$ be a semimartingale of the form

$$
d_{t} h_{t}=\left(h^{1}\right)_{\alpha}(m(t), q(t)) \xi_{k}^{\alpha} \circ d W_{t}^{k}+h^{2}(m(t), q(t)) d t,
$$

where $\left(h^{1}\right)_{\alpha}, h^{2}$ are (deterministic) smooth functions evaluated on $\left(\mathfrak{g}^{*} \times Q\right)$-valued semimartingales $(m(t), q(t))$. Similarly, denote by $\widetilde{h}_{t}$ a semimartingale of the form

$$
d_{t} \widetilde{h}_{t}=\left(\widetilde{h}^{1}\right)_{\alpha}\left(m(t), p_{q}(t)\right) \xi_{k}^{\alpha} \circ d W_{t}^{k}+\widetilde{h}^{2}\left(m(t), p_{q}(t)\right) d t,
$$

where $\left(\widetilde{h}^{1}\right)_{\alpha}, \widetilde{h}^{2} \in C^{\infty}\left(\mathfrak{g}^{*} \times T^{*} Q\right)$ are evaluated on $\left(\mathfrak{g}^{*} \times T^{*} Q\right)$-valued semimartingales $\left(m(t), p_{q}(t)\right)$. Consider the Poisson brackets defined in (4.7) and (4.8). According to Definition 13, the corresponding stochastic Hamilton 
equations on $\mathfrak{g}^{*} \times Q$ are defined to be

$$
d_{t} f(m(t), q(t))=\left\{f(m(t), q(t)), \circ d_{t} h\right\}_{\mathfrak{g}_{-}^{*} \times Q},
$$

for any $f \in C^{\infty}\left(\mathfrak{g}^{*} \times Q\right)$, respectively on $\mathfrak{g}^{*} \times T^{*} Q$,

$$
d_{t} \tilde{f}\left(m(t), p_{q}(t)\right)=\left\{\tilde{f}\left(m(t), p_{q}(t)\right), \circ d_{t} \tilde{h}\right\}_{\mathfrak{g}_{-}^{*} \times T^{*} Q},
$$

for any $\tilde{f} \in C^{\infty}\left(\mathfrak{g}^{*} \times T^{*} Q\right)$. Notice that, by the form of the Hamiltonian functionals $h$ and $\tilde{h}$, these equations are equivalent, respectively, to

$$
\begin{aligned}
d_{t} f(m(t), q(t))=\{ & \left.f(m(t), q(t)),\left(h^{1}\right)_{\alpha}(m(t), q(t))\right\}_{\mathfrak{g}_{-}^{*} \times Q} \xi_{k}^{\alpha} \circ d W_{t}^{k}+\left\{f(m(t), q(t)), h^{2}(m(t), q(t))\right\}_{\mathfrak{g}_{-}^{*} \times Q} d t \\
d_{t} \widetilde{f}\left(m(t), \boldsymbol{p}_{q}(t)\right)=\left\{\widetilde{f}\left(m(t), \boldsymbol{p}_{q}(t)\right),\left(\widetilde{h}^{1}\right)_{\alpha}\left(m(t), \boldsymbol{p}_{q}(t)\right)\right\}_{\mathfrak{g}_{-}^{*} \times T^{*} Q} \xi_{k}^{\alpha} \circ d W_{t}^{k} & \\
& +\left\{\widetilde{f}\left(m(t), \boldsymbol{p}_{q}(t)\right), \widetilde{h}^{2}\left(m(t), \boldsymbol{p}_{q}(t)\right)\right\}_{\mathfrak{g}_{-}^{*} \times T^{*} Q} d t .
\end{aligned}
$$

We now define the right hand sides of these equations involving the Poisson bracket.

For $f \in C^{\infty}\left(\mathfrak{g}^{*} \times Q\right)$, the Poisson bracket (4.7) of the two semimartingales $f(m(t), q(t))$ and $h_{t}$ then reads

$$
\begin{aligned}
\left\{f(m(t), q(t)), \circ d_{t} h_{t}\right\}_{\mathfrak{g}_{-}^{*} \times Q}:=\left[\begin{array}{c}
\frac{\partial f}{\partial m_{\alpha}}(m(t), q(t)) \\
\frac{\partial f}{\partial q^{i}}(m(t), q(t))
\end{array}\right]^{\top}\left[\begin{array}{cc}
-c_{\alpha \beta} m_{\gamma}(t) & -A_{\alpha}^{j}(q(t)) \\
A_{\beta}^{i}(q(t)) & 0
\end{array}\right]\left[\begin{array}{c}
\circ d_{t}\left(\frac{\partial h_{t}}{\partial m_{\beta}}\right) \\
\circ d_{t}\left(\frac{\partial h_{t}}{\partial q^{j}}\right)
\end{array}\right] \\
=\left\{f^{q}(m(t), q(t)), \circ d_{t} \boldsymbol{h}_{t}^{q}\right\}_{-}+\left\langle\mathbf{d} f^{m}(m(t), q(t)),\left(\circ d_{t}\left(\frac{\delta h_{t}^{q}}{\delta m}\right)\right)_{Q}(m(t), q(t))\right\rangle_{Q} \\
-\left\langle\circ d_{t} \mathbf{d} h_{t}^{m}(m(t), q(t)),\left(\frac{\delta f^{q}}{\delta m}\right)_{Q}(m(t), q(t))\right\rangle_{Q} .
\end{aligned}
$$

In this formula, $\partial f / \partial m_{\alpha}$ and $\partial f / \partial q^{i}$ are evaluated on the semimartingales $m(t)$ and $q(t)$ and, according to (2.28) and (2.29),

$$
\begin{aligned}
& d_{t}\left(\frac{\partial h_{t}}{\partial m_{\beta}}\right):=\frac{\partial\left(h^{1}\right)_{\alpha}}{\partial m_{\beta}}(m(t), q(t)) \xi_{k}^{\alpha} \circ d W_{t}^{k}+\frac{\partial h^{2}}{\partial m_{\beta}}(m(t), q(t)) d t \\
& d_{t}\left(\frac{\partial h_{t}}{\partial q^{j}}\right):=\frac{\partial\left(h^{1}\right)_{\alpha}}{\partial q^{j}}(m(t), q(t)) \xi_{k}^{\alpha} \circ d W_{t}^{k}+\frac{\partial h^{2}}{\partial q^{j}}(m(t), q(t)) d t
\end{aligned}
$$

$\mathbf{d}\left(h^{1}\right)_{\alpha}^{m}(m(t), q(t)), \mathbf{d}\left(h^{2}\right)^{m}(m(t), q(t)), \mathbf{d} f^{m}(m(t), q(t)), \frac{\delta\left(h^{1}\right)_{\alpha}^{q}}{\delta m}(m(t), q(t)), \frac{\delta\left(h^{2}\right)^{q}}{\delta m}(m(t), q(t)), \frac{\delta f^{q}}{\delta m}(m(t), q(t))$, and $\left(\frac{\delta\left(h^{1}\right)_{\alpha}^{q}}{\delta m}\right)_{Q}(m(t), q(t)),\left(\frac{\delta\left(h^{2}\right)^{q}}{\delta m}\right)_{Q}(m(t), q(t)),\left(\frac{\delta f^{q}}{\delta m}\right)_{Q}(m(t), q(t))$ are the covectors $\mathbf{d}\left(h^{1}\right)_{\alpha}^{m}(q), \mathbf{d}\left(h^{2}\right)^{m}(q)$, $\mathbf{d} f^{m}(q) \in T_{q}^{*} Q$, the elements $\delta\left(h^{1}\right)_{\alpha}^{q} / \delta m, \delta\left(h^{2}\right)^{q} / \delta m, \delta f^{q} / \delta m \in \mathfrak{g}$, and the tangent vectors $\left(\delta\left(h^{1}\right)_{\alpha}^{q} / \delta m\right)_{Q}(q)$, $\left(\delta\left(h^{2}\right)^{q} / \delta m\right)_{Q}(q),\left(\delta f^{q} / \delta m\right)_{Q}(q) \in T_{q} Q$ with the variables $(m, q)$ replaced by the semimartingales $(m(t), q(t))$,

$$
\begin{aligned}
d_{t} \mathbf{d} h_{t}^{m}(m(t), q(t)):= & \mathbf{d}\left(h^{1}\right)_{\alpha}^{m}(m(t), q(t)) \xi_{k}^{\alpha} \circ d W_{t}^{k}+\mathbf{d}\left(h^{2}\right)^{m}(m(t), q(t)) d t \\
\left(\circ d\left(\frac{\delta h_{t}^{q}}{\delta m}\right)\right)_{Q}(m(t), q(t)):= & \left(\frac{\delta\left(h^{1}\right)_{\alpha}^{q}}{\delta m}\right)_{Q}(m(t), q(t)) \xi_{k}^{\alpha} \circ d W_{t}^{k}+\left(\frac{\delta\left(h^{2}\right)^{q}}{\delta m}\right)_{Q}(m(t), q(t)) d t \\
\left\{f^{q}(m(t), q(t)), d_{t} h_{t}^{q}\right\}_{-}:=\{ & \left\{f^{q}(m(t), q(t)),\left(h^{1}\right)_{\alpha}^{q}(m(t), q(t))\right\}_{-} \xi_{k}^{\alpha} \circ d W_{t}^{k} \\
& +\left\{f^{q}(m(t), q(t)),\left(h^{2}\right)^{q}(m(t), q(t))\right\}_{-} d t .
\end{aligned}
$$


Similarly, for $\tilde{f} \in C^{\infty}\left(\mathfrak{g}^{*} \times T^{*} Q\right)$, the Poisson bracket (4.8) computed for these semimartingales is given by

$$
\begin{aligned}
& \left\{\tilde{f}\left(m(t), p_{q}(t)\right), \circ d \widetilde{h}_{t}\right\}_{\mathfrak{g}_{-}^{*} \times T^{*} Q} \\
& :=\left[\begin{array}{c}
\frac{\partial \widetilde{f}}{\partial m_{\alpha}}\left(m(t), p_{q}(t)\right) \\
\frac{\partial \widetilde{f}}{\partial q^{i}}\left(m(t), p_{q}(t)\right) \\
\frac{\partial \widetilde{f}}{\partial p_{i}}\left(m(t), p_{q}(t)\right)
\end{array}\right]^{\top}\left[\begin{array}{ccc}
-c_{\alpha \beta} m_{\gamma}(t) & -A_{\alpha}^{j}(q(t)) & p_{k}(t) \frac{\partial A_{\alpha}^{k}}{\partial q^{j}}(q(t)) \\
A_{\beta}^{i}(q(t)) & 0 & \delta_{j}^{i} \\
-p_{k}(t) \frac{\partial A_{\beta}^{k}}{\partial q^{i}}(q(t)) & -\delta_{i}^{j} & 0
\end{array}\right]\left[\begin{array}{c}
\circ d_{t}\left(\frac{\partial \widetilde{h}_{t}}{\partial m_{\beta}}\right) \\
\circ d_{t}\left(\frac{\partial \widetilde{h}_{t}}{\partial q^{j}}\right) \\
\circ d_{t}\left(\frac{\partial \widetilde{h}_{t}}{\partial p_{j}}\right)
\end{array}\right] \\
& =\left\{\tilde{f}^{p_{q}}\left(m(t), p_{q}(t)\right), \circ d_{t} \widetilde{h}_{t}^{p_{q}}\right\}_{-}+\left\langle\mathbf{d} f^{m}\left(m(t), p_{q}(t)\right),\left(\circ d_{t}\left(\frac{\delta \widetilde{h}_{t}^{p_{q}}}{\delta m}\right)\right)_{T^{*} Q}\left(m(t), p_{q}(t)\right)\right\rangle_{Q} \\
& -\left\langle\circ d_{t} \mathbf{d} \tilde{h}_{t}^{m}\left(m(t), p_{q}(t)\right),\left(\frac{\delta \tilde{f}^{p_{q}}}{\delta m}\right)_{T^{*} Q}\left(m(t), p_{q}(t)\right)\right\rangle_{Q}+\left\{\tilde{f}^{m}\left(m(t), p_{q}(t)\right), \circ d_{t} \tilde{h}_{t}^{m}\right\}
\end{aligned}
$$

with the same notational conventions as for the bracket (4.23) and where the last Poisson bracket of semimartingales is defined in (4.19).

Since $d_{t} f(m(t), q(t))=\left\langle\frac{\delta f^{q}}{\delta m}(m(t), q(t)), \circ d_{t} m(t)\right\rangle_{\mathfrak{g}}+\left\langle\mathbf{d} f^{m}(m(t), q(t)), \circ d_{t} q(t)\right\rangle_{Q}$, the stochastic Hamilton equations (i.e., the stochastic versions of equations (4.10) and (4.9)) are, respectively, the stochastic Hamel equations

$$
\begin{array}{ll}
d_{t} m(t)=\underset{\circ d_{t}\left(\frac{\delta k_{t}^{q}}{\delta m}(m(t), q(t))\right)}{\operatorname{ad}^{*} m(t)-\mathbf{J}_{T^{*} Q}\left(\circ d_{t} \mathbf{d} h_{t}^{m}(m(t), q(t))\right),} \quad d_{t} q(t)=\left(\circ d_{t}\left(\frac{\delta h_{t}^{q}}{\delta m}\right)\right)(m(t), q(t)) \quad \Longleftrightarrow \\
d_{t} m_{\alpha}(t)=-c_{\alpha \beta} \gamma^{\gamma} m_{\gamma}(t) \circ d_{t}\left(\frac{\partial h_{t}}{\partial m_{\beta}}\right)-A_{\alpha}^{j}(q(t)) \circ d_{t}\left(\frac{\partial h_{t}}{\partial q^{j}}\right), & d_{t} q^{i}(t)=A_{\beta}^{i}(q(t)) \circ d_{t}\left(\frac{\partial h_{t}}{\partial m_{\beta}}\right)
\end{array}
$$

and

$$
\begin{aligned}
& d_{t} m_{\alpha}(t)=-c_{\alpha \beta}{ }^{\gamma} m_{\gamma}(t) \circ d_{t}\left(\frac{\partial \tilde{h}_{t}}{\partial m_{\beta}}\right)-A_{\alpha}^{j}(q(t)) \circ d_{t}\left(\frac{\partial \tilde{h}_{t}}{\partial q^{j}}\right)+p_{k}(t) \frac{\partial A_{\alpha}^{k}}{\partial q^{j}}(q(t)) \circ d_{t}\left(\frac{\partial \tilde{h}_{t}}{\partial p_{j}}\right), \\
& d_{t} q^{i}(t)=A_{\beta}^{i}(q(t)) \circ d_{t}\left(\frac{\partial \tilde{h}_{t}}{\partial m_{\beta}}\right)+d_{t}\left(\frac{\partial \tilde{h}_{t}}{\partial p_{i}}\right), \quad d_{t} p_{i}(t)=-p_{k}(t) \frac{\partial A_{\beta}^{k}}{\partial q^{i}}(q(t)) \circ d_{t}\left(\frac{\partial \tilde{h}_{t}}{\partial m_{\beta}}\right)-d_{t}\left(\frac{\partial \tilde{h}_{t}}{\partial q^{i}}\right) .
\end{aligned}
$$

The last equations can be written intrinsically as

$$
\begin{aligned}
& d_{t} m(t)=\operatorname{ad}_{\circ d_{t}\left(\frac{\delta \tilde{\hbar}_{t}^{q}}{\delta m}\right)(m(t), q(t))} m(t)-\mathbf{J}_{T^{*}\left(T^{*} Q\right)}\left(o d_{t}\left(\mathbf{d} \tilde{h}_{t}^{m}\left(m(t), p_{q}(t)\right)\right)\right), \\
& d_{t} \boldsymbol{p}_{q}(t)=\left(\circ d_{t}\left(\frac{\delta \widetilde{h}_{t}^{p_{q}}}{\delta m}\right)\right)_{T^{*} Q}\left(m(t), \boldsymbol{p}_{q}(t)\right)+X_{\circ d_{t} \widetilde{h}_{t}^{m}}^{T^{*} Q}\left(m(t), p_{q}(t)\right) .
\end{aligned}
$$

By repeating the arguments in the proof of Corollary 11, we can derive the following stochastic Hamilton equations (with $P=T^{*} Q$ ) on $\mathfrak{g}^{*} \times T^{*} Q$ for these type of Hamiltonian functionals:

$$
\begin{aligned}
& d_{t} m(t)=\underset{\circ d_{t}\left(\frac{\delta \hbar_{t}^{q}}{\delta m}\right)(m(t), q(t))}{\operatorname{ad}^{*}} m(t)-\mathbf{J}_{T^{*} Q}\left(\circ d_{t} \mathbf{d} h_{t}^{m}(m(t), q(t))\right), \\
& d_{t} \boldsymbol{p}_{q}(t)=\left(d_{t}\left(\frac{\delta h_{t}^{q}}{\delta m}\right)\right)_{T^{*} Q}\left(m(t), p_{q}(t)\right)+T_{d_{t} \mathbf{d} \hbar_{t}^{m}\left(m(t), p_{q}(t)\right)}\left(m(t), p_{q}(t)\right),
\end{aligned}
$$

where

$$
T_{d_{t} \mathbf{d} h_{t}^{m}\left(m(t), p_{q}(t)\right)}\left(p_{q}\right)=\left.\frac{d}{d \epsilon}\right|_{\epsilon=0}\left(d_{t} p_{q}-\epsilon d_{t} \mathbf{d} h_{t}^{m}\left(m(t), p_{q}(t)\right)\right)
$$

in which the limit is taken in $L^{2}(\Omega)$. 
In addition, we have the non-homogeneous stochastic Lie-Poisson equations

$$
d_{t} \mathbf{J}_{T^{*} Q}\left(p_{q}(t)\right)=\underset{\circ d_{t}\left(\frac{\delta \hbar_{t}^{q(t)}}{\delta m(t)}\right)}{\operatorname{ad}_{(m(t), q(t))}} \mathbf{J}_{T^{*} Q}\left(p_{q}(t)\right)-\mathbf{J}_{T^{*} Q}\left(\circ d_{t} \mathbf{d} h_{t}^{m(t)}(m(t), q(t))\right.
$$

for $\mathbf{J}_{T * Q}\left(R_{q}(t)\right)$, where $(m(t), q(t))$ is the solution of the stochastic Hamel equations (4.25).

Remark 14. [The analog of the stochastic rigid body] In particular, the stochastic free rigid body dynamics on $\mathfrak{g}^{*}$ is obtained from (4.22) by assuming that the Hamiltonian $h: \mathfrak{g}^{*} \times Q \rightarrow \mathbb{R}$ is of the form $h(m, q):=\frac{1}{2} m_{\alpha} K^{\alpha \beta} m_{\beta}$, where $K^{\alpha \beta}$ are the components of the inner product on $\mathfrak{g}^{*}$ induced by an inner product on $\mathfrak{g}$. This Hamiltonian $h$ is computed on semimartingales of the form $d_{t} m_{\alpha}=\gamma_{\alpha} d t+\left(\Phi_{k}\right)_{\alpha} \circ d W_{t}^{k}$ with $\left(\Phi_{k}\right)_{\alpha} K^{\alpha \beta}=\xi_{k}^{\beta}$ (notice that $K:=\left[K^{\alpha \beta}\right]$ is an invertible matrix). Define $u^{\beta}:=r_{\alpha} K^{\alpha \beta}$. In particular, $\frac{\partial h}{\partial q^{2}}=0$ and $d\left(\frac{\partial h}{\partial m_{\beta}}\right)=r_{\alpha} K^{\alpha \beta} d t+\left(\Phi_{k}\right)_{\alpha} K^{\alpha \beta} \circ d W_{t}^{k}=$ $u^{\beta} d t+\xi_{k}^{\beta} \circ d W_{t}^{k}$. Thus, from (4.22), the stochastic free rigid body equations emerge as

$$
d m_{\alpha}=\left\{m_{\alpha}, m_{\beta}\right\}\left(u^{\beta} d t+\xi_{k}^{\beta} \circ d W_{t}^{k}\right)=-c_{\alpha \beta}^{\gamma} m_{\gamma} d x_{t}^{\beta} .
$$

\subsection{The Kolmogorov equations}

We start from the equations (4.21) for the Poisson manifold valued stochastic process $p(t)$ written in coordinates, namely,

$$
d_{t} R^{i}(t)=\left\{p^{i}, \phi_{\alpha}\right\}(R(t)) \xi_{k}^{\alpha} \circ d W_{t}^{k}+\left\{p^{i}, \psi\right\}(R(t)) d t .
$$

Theorem 15. The generator of the process $p(t)$ is the operator

$$
L f:=\{f, \psi\}+\frac{1}{2} \sum_{k}\left\{\phi_{\alpha} \xi_{k}^{\alpha},\left\{\phi_{\beta} \xi_{k}^{\beta}, f\right\}\right\} .
$$

Proof. We first compute the difference between the Itô and the Stratonovich differential in the process $p(t)$. Since $\left\{p^{i}, \phi_{\alpha}\right\}(p(t))=\Pi^{i j}(p(t)) \frac{\partial \phi_{\alpha}}{\partial p^{j}}(p(t))$, this difference is equal to

$$
\frac{1}{2}\left(\frac{\partial \phi_{\alpha}}{\partial p^{j}} \frac{\partial \Pi^{i j}}{\partial p^{l}}+\Pi^{i j} \frac{\partial^{2} \phi_{\alpha}}{\partial p^{l} \partial p^{j}}\right)(p(t)) \Pi^{l m}(p(t)) \frac{\partial \phi_{\beta}}{\partial p^{m}}(p(t))\left(\sum_{k} \xi_{k}^{\alpha} \xi_{k}^{\beta}\right) d t=: B^{i}(p(t)) d t .
$$

The generator then reads

$$
L f(p)=\frac{1}{2}\left(\Pi^{i m} \Pi^{j n} \frac{\partial \phi_{\alpha}}{\partial p^{m}} \frac{\partial \phi_{\beta}}{\partial p^{n}}\right)(p)\left(\sum_{k} \xi_{k}^{\alpha} \xi_{k}^{\beta}\right) \frac{\partial^{2} f}{\partial p^{i} \partial p^{j}}+\left(\Pi^{i j} \frac{\partial \psi}{\partial p^{j}}+B^{i}\right)(p) \frac{\partial f}{\partial p^{i}}
$$

which is precisely the expression 44.31).

Defining $\rho(t, p):=\mathbb{E}_{p}(f(p(t)))$ where $p(0)=p$, the function $\rho$ satisfies Kolmogorov's backward equation, namely,

$$
\frac{\partial \rho}{\partial t}=L \rho, \quad \rho(0, p)=f(p) .
$$

If the generator $L$ is a hypoelliptic operator then there exists a probability density function $\tilde{\rho}\left(t, p, p^{\prime}\right)$, defined by $\mathbb{E}_{p}(f(p(t)))=\int_{P} f\left(p^{\prime}\right) \tilde{\rho}\left(t, p, p^{\prime}\right) d p^{\prime}$; here we assume that the Poisson manifold $P$ has a volume form $d p$ relative to which this integration is carried out. This function satisfies the forward Kolmogorov (or Fokker-Planck) equation:

$$
\frac{\partial \tilde{\rho}}{\partial t}\left(t, p, p^{\prime}\right)=L_{p^{\prime}}^{*} \tilde{\rho}\left(t, p, p^{\prime}\right)
$$

with $\tilde{\rho}\left(0, p, p^{\prime}\right)$ equal to the Dirac measure $\delta\left(p^{\prime}-p\right)$ and where $L^{*}$ denotes the adjoint of $L$.

Next, we give a sufficient condition, in terms of the measure on $P$ used to define the probability density function $\rho\left(t, p, p^{\prime}\right)$, ensuring a nice formula for the formal adjoint of the operator $L$ defined in (4.31). We take a measure 
on $P$ which is induced by a volume form $\Lambda \in \Omega^{\operatorname{dim} P}(P)$. We say that a volume form $\Lambda$ on $P$ is Hamiltonian, if $0=£_{X_{g}} \Lambda=\operatorname{di}_{X_{g}} \Lambda+\mathbf{i}_{X_{g}} \mathbf{d} \Lambda=\mathbf{d i}_{X_{g}} \Lambda$, for all $g \in C^{\infty}(P)$. Therefore

$$
\begin{aligned}
\operatorname{div}\left(f X_{g}\right) \Lambda & =£_{f X_{g}} \Lambda=\mathbf{i}_{f X_{g}} \mathbf{d} \Lambda+\mathbf{d i}_{f X_{g}} \Lambda=\mathbf{d}\left(f \mathbf{i}_{X_{g}} \Lambda\right) \\
& =\mathbf{d} f \wedge \mathbf{i}_{X_{g}} \Lambda+f \mathbf{d} \mathbf{i}_{X_{g}} \Lambda=-\mathbf{i}_{X_{g}}(\mathbf{d} f \wedge \Lambda)+\left(\mathbf{i}_{X_{g}} \mathbf{d} f\right) \Lambda \\
& =X_{g}[f] \Lambda=\{f, g\} \Lambda .
\end{aligned}
$$

This shows that $\operatorname{div}\left(f X_{g}\right)=\{f, g\}$ for any $f, g \in C^{\infty}(P)$.

Hence, by the Stokes Theorem,

$$
\int_{P}\{f, g\} \Lambda=\int_{P} \operatorname{div}\left(f X_{g}\right) \Lambda=\int_{P} \operatorname{di}_{f X_{g}} \Lambda=\int_{\partial P} \mathbf{i}_{f X_{g}} \Lambda=\int_{\partial P} f \mathbf{i}_{X_{g}} \Lambda=-\int_{\partial P} g \mathbf{i}_{X_{f}} \Lambda ;
$$

in which the last equality follows by skew-symmetry of the Poisson bracket.

Now let $f, g, h \in C^{\infty}(P)$ and integrate the identity $\{h f, g\}=h\{f, g\}+f\{h, g\}$ to get

$$
\int_{P} h\{f, g\} \Lambda+\int_{P} f\{h, g\} \Lambda=\int_{P}\{h f, g\} \Lambda .
$$

By (4.34), the term on the right hand side vanishes if $\partial P=\varnothing$ or if at least one of $f$ or $g$ vanish on $\partial P$. In these cases, we have

$$
\int_{P}\{f, g\} h \Lambda=\int_{P} f\{g, h\} \Lambda
$$

If $(P, \omega)$ is a $2 n$-dimensional symplectic manifold, the Liouville volume $\Lambda:=\frac{(-1)^{n(n-1) / 2}}{n !} \omega \wedge \ldots \wedge \omega(n$ times $)$ is Hamiltonian. Indeed, since $£_{X_{g}} \omega=0$ for any $g \in C^{\infty}(P)$, it immediately follows that $£_{X_{g}} \Lambda=0$.

Corollary 16. Let $(P,\{\cdot, \cdot\})$ be a boundaryless Poisson manifold and $\Lambda$ a Hamiltonian volume form on P. Relative to the $L^{2}$-inner product on $P$ defined by $\Lambda$, the formal adjoint of the linear operator $L$ defined in (4.31) may be expressed as

$$
L^{*} f=-\{f, \psi\}+\frac{1}{2} \sum_{k}\left\{\phi_{\beta} \xi_{k}^{\beta},\left\{\phi_{\alpha} \xi_{k}^{\alpha}, f\right\}\right\}
$$

This corollary follows directly from (4.31) and 4.35).

Consider the Poisson manifold $\mathfrak{g}^{*} \times Q$ and the stochastic Hamiltonian (2.27). Define the semimartingale $u(m(q(t), p(t)), q(t))$, where $u \in C^{\infty}\left(\mathfrak{g}^{*} \times Q\right)$. In this case, Kolmogorov's backward equation for $\rho(t, m, q):=$ $\left.\mathbb{E}_{(m, q)}(f(m(q(t), p(t))), q(t))\right)$ takes the form

$$
\frac{\partial \rho}{\partial t}=\left\{\rho, m_{\alpha} u^{\alpha}-\ell(u, q)\right\}+\frac{1}{2} \sum_{k}\left\{m_{\alpha} \xi_{k}^{\alpha},\left\{m_{\beta} \xi_{k}^{\beta}, \rho\right\}\right\}
$$

with $\rho(0, m, q)=f(m, q)$.

Now choose a Hamiltonian volume form $\Lambda$ on the Poisson manifold $\mathfrak{g}^{*} \times T^{*} Q$. Using the measure defined by $\Lambda$, and computing the formal adjoint of $L$ (the right hand side of (4.37) ) given by (4.36), we get Kolmogorov's forward, or Fokker-Planck, equation

$$
\frac{\partial \tilde{\rho}}{\partial t}=-\left\{\tilde{\rho}, m_{\alpha} u^{\alpha}-\ell(u, q)\right\}+\frac{1}{2} \sum_{k}\left\{m_{\alpha} \xi_{k}^{\alpha},\left\{m_{\beta} \xi_{k}^{\beta}, \tilde{\rho}\right\}\right\}
$$

with $\tilde{\rho}\left(0,(m, q),\left(m^{\prime}, q^{\prime}\right)\right)=\delta\left(\left(m^{\prime}, q^{\prime}\right)-(m, q)\right)$.

Remark 17. Assume we work on $\mathfrak{g}^{*}$, where $\mathfrak{g}$ is a compact Lie algebra, for simplicity. Then there is an invariant inner product on $\mathfrak{g}$ and, using it, we define an invariant inner product on $\mathfrak{g}^{*}$ whose norm is denoted by $\|\cdot\|$. In this case, $m \mapsto\|m\|^{2}$ is a Casimir function. As Casimirs are conserved along the stochastic flows of the stochastic Hamiltonian semimartingales, we have $d_{t}\|m\|^{2}(q(t), p(t))=0$. As a consequence, there exists (cf. [25]) an invariant probability measure $\mu$ on $\mathfrak{g}^{*} \times Q$ for the motion. Namely, the measure satisfies

$$
\left.\int \mathbb{P}_{(m, q)}(m(q(t), p(t)), q(t)) \in B\right) d \mu(m, q)=\mu(B),
$$

for all Borel sets $B \subset \mathfrak{g}^{*} \times Q$. This measure disintegrates along the level sets of the Casimir $\|m\|^{2}$. 


\section{Acknowledgements}

We are enormously grateful to our colleagues for their helpful encouraging remarks and interesting enjoyable discussions: A. Arnaudon, M. Arnaudon, S. Albeverio, J.-M. Bismut, N. Bou-Rabee, A. L. Castro, M. D. Chekroun, G. Chirikjian, D. O. Crisan, J. Eldering, M. Engel, F. Gay-Balmaz, A. Grandchamp, P. Lynch, J.-P. Ortega, G. Pavliotis, V. Putkaradze, and C. Tronci. We also acknowledge the Bernoulli Center at EPFL where parts of this work were initiated. ABC was partially supported by Portuguese FCT grant SFRH/BSAB/105789/2014, DDH by ERC Advanced Grant 267382 FCCA as well as EPSRC Grant EP/N023781/1, and TSR by NCCR SwissMAP grant of the Swiss NSF.

\section{References}

[1] A. Arnaudon, A.L. Castro, D.D. Holm [2016] Noise and dissipation on coadjoint orbits, J. Nonlin. Sci. DOI 10.1007/s00332-017-9404-3. Preprint at http://arxiv.org/pdf/1601.02249.pdf

[2] A. Arnaudon, A.L. Castro, D.D. Holm [2016] Noise and dissipation in rigid body motion, http://arxiv.org/pdf/1606.06308.pdf

[3] M. Arnaudon, X. Chen, A.B. Cruzeiro [2014] Stochastic Euler-Poincaré reduction, J. Math. Physics 55, 081507.

[4] J.-M. Bismut [1981] Mécanique aléatoire, Lect. Notes Math., 866, Springer-Verlag, Berlin, New York (1981).

[5] J.-M. Bismut [1984] Large Deviations and the Malliavin Calculus, Progress in Mathem., 45, Birkhüser Boston, Inc., 1984.

[6] A. M. Bloch [2015] Nonholonomic Mechanics and Control, second edition. With the collaboration of J. Bailieul, P.E. Crouch, J.E. Marsden, D. Zenkov. With scientific input from P.S. Krishnaprasad and R.M. Murray. Interdisciplinary Applied Mathematics, 24, Springer-Verlag, New York (2015).

[7] A.M. Bloch, J.E. Marsden, D.V. Zenkov [2009] Quasivelocities and symmetries in non-holonomic systems, Dyn. Syst., 24(2), 187-222.

[8] X. Chen, A.B. Cruzeiro, T.S. Ratiu [2015] Stochastic variational principles for dissipative equations with advected quantities; http://arxiv.org/abs/1506.05024

[9] A. Clebsch [1859] Über die Integration der hydrodynamischen Gleichungen. J. reine angew. Math., 56, 1-10.

[10] A.B. Cruzeiro, P. Malliavin [1998] Non perturbative construction of invariant measure through confinement by curvature. J. Math. Pures Appl., 77, 527-537.

[11] B.K. Driver [1994] A Cameron-Martin type quasi-invariance theorem for pinned Brownian motion on a compact Riemannian manifold. Trans. Amer. Math. Soc., 342(1), 375-395.

[12] K.D. Elworthy, X-M. Li [2006] Geometric stochastic analysis on path spaces. I Int. Congress of Math., Vol. III, Eur. Math. Soc., Zürich, 575-594.

[13] F. Gay-Balmaz, T.S. Ratiu [2011] Clebsch optimal control formulation in mechanics, J. Geom. Mech., 3(1), $41-79$.

[14] V. Guillemin, S. Sternberg [1980] The moment map and collective motion, Ann. Phys., 127(1), 220-253.

[15] G. Hamel [1904] Die Lagrange-Eulersche Gleichungen der Mechanik, Z. Math. Phys., 50, 1-57.

[16] S. Hochgerner, T.S. Ratiu [2015] Geometry of non-holonomic diffusion, J. Eur. Math. Soc. (JEMS), 17(2), 273-319.

[17] D.D. Holm [2015] Variational principles for stochastic fluid dynamics, Proc. Roy. Soc. A, 471, no. 2176, 20140963, 19pp; http://rspa.royalsocietypublishing.org/content/471/2176/20140963. 
[18] D.D. Holm, T. Schmah, and C. Stoica. Geometry, Symmetry and Mechanics. Oxford University Press, 2009.

[19] N. Ikeda, S. Watanabe [1989] Stochastic Differential Equations and Diffusion Processes, North Holland/Kodansha (1989).

[20] P.S. Krishnaprasad, J.E. Marsden [1987] Hamiltonian structures and stability for rigid bodies with flexible attachments, Arch. Rat. Mech. Anal., 98(1), 71-93.

[21] J.A. Lázaro-Camí, J.-P. Ortega [2008] Stochastic Hamiltonian dynamical systems, Rep. Math. Phys., 61(1), $65-122$.

[22] P. Malliavin [1997] Stochastic Analysis, Grund. der mathem. Wissen., 313, Springer-Verlag, New York (1997).

[23] C.-M. Marle [2013] On Henri Poincaré's note "Sur une forme nouvelle des équations de la Mécanique", J. Geom. Symmetry Phys., 29, 1-38.

[24] J.E. Marsden, T.S. Ratiu [1999] Introduction to Mechanics and Symmetry, second edition, Texts Appl. Math., 17, Springer-Verlag, New York (1999).

[25] Y. Miyahara [1973] Invariant measures of ultimately bounded stochastic processes, Nagoya Math. J., 49, 149153.

[26] J.-P. Ortega, T.S. Ratiu [2004] Momentum Maps and Hamiltonian Reduction, Progress in Mathematics, 222, Birkhüser Boston, Inc., 2004.

[27] H. Poincaré [1901] Sur une forme nouvelle des équations de la mécanique, C.R. Acad. Sci., 132, 369-371. 